Die Eurokrise: Ein Quantensprung in der Politisierung des europäischen Integrationsprozesses?

To be published in: PVS 2015

Prof. Dr. Edgar Grande

Lehrstuhl für Vergleichende Politikwissenschaft

Ludwig-Maximilians-Universität München

Geschwister-Scholl-Institut für Politikwissenschaft

Oettingenstr. 67

80538 München

E-Mail: grande@lrz.uni-muenchen.de

Prof. Dr. Hanspeter Kriesi

Stein Rokkan Chair of Comparative Politics

European University Institute

Via dei Roccettini, 9

50014 San Domenico di Fiesole

Florenz/Italien

Email: Hanspeter.Kriesi@eui.eu 


\title{
Zusammenfassung
}

Dieser Beitrag untersucht die Auswirkungen der Eurokrise auf die Politisierung Europas. Im Mittelpunkt der Analyse steht die Frage, ob die Eurokrise im Vergleich zu früheren Integrationsdebatten einen Quantensprung in der Politisierung des europäischen Integrationsprozesses bewirkte. Um diese Frage zu beantworten, wird die öffentliche Debatte zur Eurokrise mit den Integrationsdebatten der vergangenen vierzig Jahre verglichen. Unsere Untersuchung umfasst sechs westeuropäische Länder (Deutschland, Frankreich, Großbritannien, Österreich, Schweden und die Schweiz), in denen alle Vertiefungsschritte (von der Einheitlichen Europäischen Akte bis zum Vertrag von Lissabon) und sämtliche Erweiterungsrunden (von der sogenannten „Norderweiterung“ 1973 bis zu den beiden „Osterweiterungen“ 2004 und 2007) erfasst wurden. Der Beitrag kommt zu dem Ergebnis, dass die Eurokrise in den untersuchten Ländern zwar politisiert hat, dass sie aber zu keinem Quantensprung im politischen Konfliktniveau führte, sondern die Dominanz der exekutiven Eliten in der öffentlichen Debatte verstärkte

Schlagwörter: Europa, EU, Eurokrise, Politisierung, Politischer Konflikt

\begin{abstract}
This article examines the impact of the Eurozone crisis on the politicization of the European integration process. Our analysis explores whether the crisis caused a quantum leap in the scope and intensity of politicization compared with previous integration debates. We compare the public debate on the Eurozone crisis with previous debates on European integration spanning the past forty years. Our study includes six West European countries (Austria, Britain, France, Germany, Sweden, and Switzerland) and it covers every major treaty reform (the Single European Act, Maastricht, Amsterdam, the Constitutional Treaty, and the Lisbon Treaty) and every enlargement debate (from the 'Northern' enlargement in 1973 to the two 'Eastern' enlargements in 2004 and 2007). The article concludes that the Eurozone crisis was politicized in the countries under scrutiny, but that it did not cause a quantum leap in the level and scope of political conflict. Rather, it reinforced the dominance of executive elites in public debate.
\end{abstract}

Keywords: Europe, EU, Eurozone crisis, politicization, political conflict 


\section{Die Eurokrise: Ein Quantensprung in der Politisierung des europäischen Integrationsprozesses?}

\section{Einleitung ${ }^{1}$}

Die Frage, ob die Eurokrise zu einer Politisierung des europäischen Integrationsprozesses geführt hat, scheint trivial zu sein. Seit dem Bekanntwerden der finanziellen Schwierigkeiten Griechenlands im Dezember 2009 bestimmte sie nicht nur in den am stärksten betroffenen „Schuldnerländern“ (Griechenland, Irland, Italien, Portugal und Spanien) die politische Agenda, sondern auch in den anderen Mitgliedstaaten der EU. Intensive öffentliche Debatten, Massenproteste, die Abwahl von Regierungen jedweder Couleur, das Erstarken rechtspopulistischer und euroskeptischer Parteien bei der Europawahl 2014 - all das scheint eindeutig zu zeigen, dass die politischen Konflikte um das „Projekt Europa“ eine neue Qualität erreicht haben. Im Vergleich zu früheren Integrationsschritten und -debatten scheint die Eurokrise einen Quantensprung in der öffentlichen Sichtbarkeit Europas (Risse 2014a, S. 2) und in seiner kritischen Wahrnehmung bewirkt zu haben.

Vor diesem Hintergrund überrascht es nicht, dass die einschlägige politikwissenschaftliche Literatur zu diesem Thema von drei Thesen beherrscht wird (Statham u. Trenz 2013a, 2014; Rauh u. Zürn 2014; Risse 2014b; Zürn u. Rauh 2014). Erstens wird angenommen, dass die Eurokrise einen „kosmopolitisches Moment“ (Beck 2000) erzeugte, durch das nationale politische Debatten auf eine einzigartige Weise integriert und europäisiert wurden (Beck 2012; Risse 2014b). Zweitens wird behauptet, dass die Eurokrise zu einem bislang beispiellosen Ausmaß an Politisierung mit einer außergewöhnlich großen Salienz und einer besonders breiten Beteiligung in öffentlichen Debatten und in Protestereignissen geführt habe (Rauh u. Zürn 2014). Dieses hohe Konfliktniveau wird schließlich, drittens, damit erklärt, dass die Politisierung der Eurokrise durch manifeste Verteilungskonflikte sowohl zwischen als auch innerhalb von Mitgliedstaaten angetrieben wurde und nicht durch abstrakte Folgeprobleme des Kompetenztransfers an die EU oder die Aufnahme neuer Mitglieder (Statham u. Trenz 2013a, S. 164). Dies führte insgesamt - so die vorherrschende Meinung -

\footnotetext{
${ }^{1}$ Dieser Beitrag basiert auf Forschungsarbeiten des Projekts „Die Politisierung Europas“, das von 2010 bis 2014 unter unserer Leitung mit Mitteln der Deutschen Forschungsgemeinschaft durchgeführt wurde. Wir sind dem gesamtem Projektteam - dem Projektkoordinator Swen Hutter, den ProjektmitarbeiterInnen Regina Becker, Daniela Braun, Silke Breimaier, Alena Kerscher und Simon Maag, unseren Kooperationspartnern Martin Dolezal (Wien) und Johan Hellstrøm (Umea), sowie zahlreichen studentischen Hilfskräften für die Kodierung der Artikel zu großem Dank verpflichtet.
} 
dazu, dass sich in der Eurokrise nicht nur das Ausmaß und die Intensität der Politisierung Europas deutlich von früheren Integrationskonflikten unterscheiden, sondern auch ihr Inhalt.

Die vorliegenden empirischen Belege für diese Thesen sind jedoch noch keineswegs überzeugend. Die umfassendste Überprüfung haben bislang Rauh und Zürn (2014) vorgenommen, die Mediendebatten und die öffentlichen Meinungen in den sechs Gründungsmitgliedstaaten der EU analysierten. Ihre Daten zeigen eine deutliche Steigerung der Politisierung seit den frühen 1990er Jahren mit einem klaren Höhepunkt in der Eurokrise. Auch die Fallstudie von Hutter und Kerscher (2014) zur Bedeutung des Europathemas in den französischen Präsidentschaftswahlen von 1974 bis 2012 ergab, dass durch die Eurokrise europäische Themen und die Bedeutung ökonomischer Frames erheblich zugenommen haben. Schließlich konnte Kriesi (2014) in seiner vergleichenden Analyse der Auswirkungen der Eurokrise auf die nationalen Wahlen in Europa zeigen, dass die amtierenden Regierungen unabhängig von ihrer parteipolitischen Zugehörigkeit abgestraft wurden. Dennoch ist die empirische Fundierung der Thesen zur Politisierung Europas in der Eurokrise insgesamt noch unzureichend und es fällt leicht, Gegenbeispiele zu finden. So spielte das Europathema bei der deutschen Bundestagswahl im September 2013, der ersten nach dem Ausbruch der Eurokrise, nur eine untergeordnete Rolle; und die Analyse von Serricchio et al. (2013) zeigt darüber hinaus, dass die globale Finanzkrise insgesamt nicht dazu geführt hat, dass ökonomische Faktoren als Quelle von Euroskepsis eine wichtigere Rolle spielen.

In diesem Beitrag sollen die vorliegenden Thesen zu den Auswirkungen der Eurokrise auf die Politisierung Europas systematisch empirisch überprüft werden. Zu diesem Zweck werden wir die öffentliche Debatte zur Eurokrise mit den Integrationsdebatten der vergangenen vierzig Jahre vergleichen. Unsere Untersuchung umfasst sechs westeuropäische Länder (Deutschland, Frankreich, Großbritannien, Österreich, Schweden und die Schweiz), in denen alle Vertiefungsschritte (von der Einheitlichen Europäischen Akte bis zum Vertrag von Lissabon) und sämtliche Erweiterungsrunden (von der sogenannten „Norderweiterung“ 1973 bis zu den beiden „Osterweiterungen“ 2004 und 2007) erfasst wurden. Im Mittelpunkt unserer Analyse stehen die folgenden Fragen: Hat die Eurokrise tatsächlich im Vergleich zu früheren Integrationsdebatten einen Quantensprung in der Politisierung des europäischen Integrationsprozesses bewirkt? Welche Akteure haben die Debatte um die Eurokrise bestimmt? Und welche Rolle spielten redistributive Konflikte und nationale Frames in dieser Debatte? 
Unser Beitrag ist in acht Teile gegliedert. Wir werden zunächst unser Konzept der Politisierung präsentieren und einige theoretische Überlegungen zur Politisierung der EuroKrise formulieren (Abschnitt 2). Danach werden wir das methodische Vorgehen unserer Untersuchung beschreiben (Abschnitt 3). In den folgenden Schritten werden die empirischen Ergebnisse der Analyse präsentiert. Wir beginnen mit einer Darstellung des Gesamtverlaufs der Politisierung in der Eurokrisen-Debatte (Abschnitt 4), werden diese Debatte dann mit früheren Integrationsdebatten vergleichen (Abschnitt 5) und Unterschiede zwischen den untersuchten Ländern herausarbeiten (Abschnitt 6). Schließlich werden wir die Akteure analysieren, die an der Debatte beteiligt waren und der Frage nachgehen, welche Rolle nationalistisch-identitäre Begründungsmuster in der Eurokrisen-Debatte spielten (Abschnitt 7). Wir kommen zu dem Ergebnis, dass die Eurokrise in den von uns untersuchten „Geberländern“ zwar politisiert hat, dass sie aber zu keinem Quantensprung im politischen Konfliktniveau führte, sondern die Dominanz der exekutiven Eliten in der öffentlichen Debatte verstärkte (Abschnitt 8).

\section{Die Politisierung der Eurokrise: Theoretisches Konzept und Hypothesen}

Was verstehen wir unter Politisierung und weshalb sollte die Eurokrise einen neuen Höhepunkt in der Politisierung des europäischen Integrationsprozesses bewirken? Der Begriff der „Politisierung“ spielt in der Politikwissenschaft in mehreren Forschungskontexten eine Rolle - von der Politischen Ökonomie über die Verwaltungswissenschaft und die politische Kommunikation bis zur Europaforschung und den Internationalen Beziehungen - und er wird darin auf unterschiedliche Weise konzeptualisiert (Hooghe u. Marks 2012; de Wilde 2011; Zürn 2012). Die folgende Analyse basiert auf einem Konzept von Politisierung, das auf die Arbeiten von Schattschneider $(1957,1960)$ zurückgeht und in dessen Mittelpunkt der politische Konflikt steht. Politisierung kann dann verstanden werden als „expansion in the scope of conflict“ (Schattschneider 1960, S. 12), sei es innerhalb des politischen Systems, sei es zwischen dem politischen System und anderen gesellschaftlichen Teilsystemen (wie der Wirtschaft). Die Ausweitung politischen Konflikts kann in mehreren Dimensionen erfolgen: seiner öffentlichen Sichtbarkeit (visibility), dem Kreis der beteiligten Akteure (scope of actors) und der Intensität der Auseinandersetzung zwischen diesen Akteuren (intensity).

Die erste Dimension, die öffentliche Sichtbarkeit bzw. Salienz, basiert auf der Annahme, dass Öffentlichkeit eine unverzichtbare Bedingung für das Austragen politischer 
Konflikte ist. Themen, die nicht öffentlich debattiert werden, können allenfalls in geringem Umfang politisiert werden. Dies gilt umso mehr, als sich moderne Demokratien zu „Mediendemokratien“ entwickelt haben, in denen die mediale Präsenz von Akteuren und Themen eine entscheidende Voraussetzung für ihre politische Relevanz ist. Im Unterschied zu Green-Pedersen (2012) und Guinaudeau und Persico (2013) gehen wir jedoch nicht davon aus, dass die öffentliche Sichtbarkeit eines Konflikts der einzige Aspekt ist, der für seine Politisierung ausschlaggebend ist. Die zweite Dimension von Politisierung ist die Ausweitung des Kreises der an einem Konflikt beteiligten Akteure. Solange Konflikte nicht über einen kleinen Kreis politischer Eliten oder interessierter Bürgergruppen hinausgehen, sind sie nur in geringem Umfang politisiert. Im Fall des Europathemas wurde in der Literatur insbesondere die dominante Stellung der Exekutiven, seien es die Regierungen der Mitgliedstaaten, seien es die Mitglieder supranationaler Institutionen, betont und für das insgesamt niedrige Politisierungsniveau verantwortlich gemacht (Koopmans 2007, 2010; Statham u. Trenz 2013b, S. 79ff.). Vor diesem Hintergrund kommt der Frage, ob die Eurokrise die Dominanz der Exekutiven gebrochen und den Konflikt um Europa in den Mittelpunkt des Parteienwettbewerbs gerückt oder zum Gegenstand zivilgesellschaftlicher Mobilisierung gemacht hat, eine entscheidende Bedeutung zu. Die dritte Dimension von Politisierung, Polarisierung, bezieht sich auf die Intensität der Auseinandersetzung zwischen den Akteuren. Ein politischer Konflikt ist umso intensiver, je stärker er zwischen Akteuren polarisiert. Stark politisierte Konflikte zeichnen sich dadurch aus, dass die beteiligten Akteure unterschiedliche Positionen vertreten und sich zu gegensätzlichen politischen Lagern formieren (de Wilde 2011; Höglinger 2012). Politische Konflikte sind folglich dann am stärksten polarisiert, wenn sich zwei politische Gruppierungen mit gegensätzlichen Positionen zu einem Thema gegenüberstehen, dem sie beide eine große Bedeutung beimessen.

Zwischen diesen Dimensionen bestehen zwar Zusammenhänge, es dürfte in der Forschung zur Politisierung Europas inzwischen jedoch weitgehend Konsens herrschen, dass sich Politisierung nicht auf eine einzige Dimension reduzieren lässt (de Wilde 2011; de Wilde u. Zürn 2012; Höglinger 2012; Statham u. Trenz 2013a; Hutter u. Grande 2014). Um dennoch quantifizierbare Aussagen über das Gesamtniveau der Politisierung machen zu können und nicht nur über einzelne Dimensionen, haben Hutter und Grande (2014) einen kombinierten Politisierungsindex entwickelt. Dieser Index berücksichtigt die große Bedeutung der Salienz, indem diese mit der Summe der beiden anderen Dimensionen multipliziert wird: Politisierung = Salienz x (Akteursexpansion + Polarisierung). Wir werden diesen Index für die weitere 
Analyse verwenden und seine Berechnung im Methodenteil (Abschnitt 3) ausführlicher erläutern.

Wenn man dieses mehrdimensionale Konzept der Politisierung auf die Debatte von Integrationskonflikten wie die Eurokrise anwendet, dann lassen sich mehrere Hypothesen zur Intensität des Konflikts um die Euro-Rettungspolitik formulieren. Die erste Hypothese zielt auf den Zusammenhang zwischen der Herausbildung einer europäischen Öffentlichkeit und der Salienz der öffentlichen Debatte um die Eurokrise ab. Die Literatur zur europäischen Öffentlichkeit nimmt an, dass die „emergence of Europeanized public spheres constitutes a first step in the politicization of European policies“ (Risse 2010, S. 232; Risse 2014b). Demnach wird die Salienz eines Themas stark von der Struktur einer öffentlichen Debatte beeinflusst, insbesondere dem Grad ihrer Europäisierung. Je stärker eine Debatte europäisiert ist, desto größer sollte ihre Salienz sein. Dabei wird keine vollständig integrierte europäische Öffentlichkeit mit eigenen Medien, Foren und Akteuren unterstellt. Koopmans und Statham (2010a, S. 38) haben stattdessen zwei weniger anspruchsvolle Formen der Europäisierung nationaler Öffentlichkeiten unterschieden: einerseits eine vertikale Europäisierung, bei der nationale Akteure entweder Themen einen europäischen Bezug geben oder europäische Akteure und Institutionen adressieren; und andererseits eine horizontale Europäisierung, bei der Akteure kommunikative Bezüge zwischen verschiedenen Ländern herstellen oder sich sogar an politischen Debatten in einem anderen Land beteiligen. In unserer empirischen Analyse der Struktur der Eurokrisen-Debatte konnten wir zeigen, dass dort beide Formen der Europäisierung zu finden sind und, dass zudem eine Parallelisierung der nationalen Debatten in der Eurokrise stattfand (Kriesi u. Grande 2014). Die Eurokrise fungierte als gemeinsamer Fokus für die verschiedenen nationalen Öffentlichkeiten, auch wenn die Debatten in den einzelnen Ländern stark von der unterschiedlichen nationalen Betroffenheit von der Krise und unterschiedlichen nationalen Problemsichten geprägt war, wie Drewski (2015) in seiner vergleichenden Analyse des öffentlichen Diskurses zur Eurokrise in Deutschland und Spanien zeigte. Dennoch können wir vor diesem Hintergrund erwarten, dass die Europäisierung der nationalen Öffentlichkeiten zu einer sehr hohen Salienz der Eurokrise in öffentlichen Debatten führte (Salienzthese).

Wir gehen zudem davon aus, dass die Salienz der Eurokrise nicht nur durch die Struktur der europäischen Öffentlichkeit beeinflusst wird, sondern auch durch die differenzierte Struktur der EU (Leuffen et al. 2013). Die EU setzt sich bekanntlich aus mehreren funktionalen Regimen mit unterschiedlicher Mitgliedschaft zusammen und die 
Eurozone ist eines dieser funktionalen Regime mit einem begrenzten Mitgliederkreis und eigenen formellen und informellen Institutionen (u.a. EZB und Euro-Gruppe). Zum Zeitpunkt der Eurokrise hatten 17 EU-Mitgliedstaaten den Euro eingeführt, von denen drei (Deutschland, Frankreich und Österreich) von unserer Untersuchung erfasst werden. Vergleichende Analysen zur Politisierung des europäischen Integrationsprozesses haben generell große Unterschiede zwischen Ländern festgestellt, wobei sich die beobachten Variationen nicht immer durch die Dauer der Mitgliedschaft oder die Stärke der Integration in die EU erklären lassen (vgl. U.a. Hutter u. Grande 2014). Dennoch ist zu erwarten, dass die Salienz der Eurokrise in den Mitgliedsstaaten der Eurozone aufgrund ihrer stärkeren Betroffenheit von der Krise größer ist als in anderen Ländern (Länderdifferenzthese). ${ }^{2}$

Auch wenn wir davon ausgehen, dass die Eurokrise, aufgrund der starken Europäisierung der nationalen Öffentlichkeiten, ein außerordentlich salientes Thema war wenngleich mit erheblichen Variationen zwischen Ländern -, so können wir doch nicht annehmen, dass sich die beiden anderen Dimensionen des Politisierungskonzepts in gleicher Weise entwickeln. Dies gilt zunächst für die Reichweite des Kreises der involvierten Akteure. Die EU ist bekanntlich durch ein System der doppelten Repräsentation mit jeweils eigenen Institutionen und eigenen Kanälen der politischen Einflussnahme gekennzeichnet: der territorialen Repräsentation der Mitgliedstaaten (durch den Europäischen Rat und den Ministerrat) und der politischen Repräsentation der europäischen Bürger (durch das Europäische Parlament) (Schimmelfennig 2010). Im Fall der EU tragen zwei Faktoren dazu bei, dass aus diesem System der doppelten Repräsentation eine spezifische Akteurskonstellation resultiert. Erstens ist die territoriale Repräsentation in der EU (wie im deutschen Föderalismus) Aufgabe der nationalen Exekutiven, insbesondere ihrer Regierungen; die Mitglieder der verschiedenen Räte werden weder von den Bürgern direkt (wie in den USA), noch von den nationalen Parlamenten (analog zur Besetzung des Bundesrates in Österreich) bestellt. Auf diese Weise erhalten die nationalen Exekutiven ein besonderes Gewicht in europäischen Entscheidungsprozessen, indem der territoriale Kanal der Einflussnahme zu einem (inter)gouvernementalen Kanal wird. Dies wird, zweitens, noch

\footnotetext{
${ }^{2}$ Unser Ländersample enthält keine sogenannten Schuldnerländer, es ist jedoch davon auszugehen, dass es sich hierbei um keine homogene Ländergruppe handelt und die Salienz der Eurokrise nicht in allen diesen Ländern höher ist als in den von uns untersuchten Geberländern. Drewskis vergleichende Analyse der Leitartikel in den großen Tageszeitungen in Deutschland und Spanien zeigt, dass die Salienz des Themas in der ersten Phase der Eurokrise (bis zum Sommer 2010) in Spanien deutlich niedriger war als in Deutschland. Erst als Spanien selbst unmittelbar betroffen war (aufgrund der finanziellen Schwierigkeiten der spanischen Bank Bankia), ist die Salienz auf das deutsche Niveau angestiegen, sie lag aber in keiner Phase höher (Drewski 2015, S. 13). Das schließt aber nicht aus, dass die Salienz des Konflikts in anderen Krisenländern, insbesondere in Griechenland, größer ist.
} 
verstärkt durch die Tatsache, dass die politische Repräsentation durch politische Parteien aufgrund der dem europäischen Mehrebenensystem inhärenten Organisationsprobleme unterentwickelt ist (Hix u. Lord 1997). Die organisatorische Basis der Parteien und der Schwerpunkt des Parteienwettbewerbs liegen nach wie vor auf der nationalen Ebene und dies hat zur Folge, dass europäische Themen im parteipolitischen Kanal unterrepräsentiert sind. ${ }^{3}$ Beides zusammengenommen bewirkt, dass im politischen System der EU nicht nur politische Entscheidungsprozesse, sondern auch die öffentlichen Debatten durch exekutive Akteure, seien es nationale Regierungen, seien es Mitglieder supranationaler Institutionen, dominiert werden (Koopmans 2010).

Die Eurokrise bildet keine Ausnahme hiervon. Im Management der Eurokrise, bei den Entscheidungen zu den einzelnen Hilfspaketen und zu den institutionellen Reformen der Wirtschafts- und Währungsunion, spielten die nationalen Regierungen die maßgebliche Rolle. Nicht von ungefähr bildeten die Gipfeltreffen der Staats- und Regierungschefs der EU die fokalen Ereignisse in der Eurokrise, in denen die öffentlichen Debatten kulminierten und die Aufmerksamkeitszyklen der Medien ihre Höhepunkte erreichten (Kriesi u. Grande 2014; Drewski 2015). Aufgrund der starken supranationalen Kompetenzen im Bereich der Geldpolitik und der Einbeziehung des Internationalen Währungsfonds (IWF) in die Hilfsmaßnahmen, haben in der Eurokrise neben supranationalen Akteuren, wie der Kommission, auch politikfeldspezifische exekutive Akteure, wie die Euro-Gruppe (das informelle Treffen der Finanzminister der Eurozone), die EZB und die „Troika“ (bestehend aus Vertretern des IWF, der Kommission und der EZB) eine wichtige Rolle gespielt. Aus all diesen Gründen erwarten wir in der Debatte um die Eurokrise eine Akteurskonstellation, die sich nicht nur in ihrer Zusammensetzung deutlich von früheren Integrationsdebatten unterscheidet, wir nehmen auch an, dass die Bedeutung exekutiver Akteure in der Eurokrise insgesamt signifikant größer ist (Exekutivdominanzthese).

Der dritte Aspekt unseres Politisierungskonzepts hängt eng mit dem zweiten, der Bedeutung exekutiver Akteure, zusammen. Wir gehen davon aus, dass die Polarisierung öffentlicher Debatten umso stärker ist, je größer die Bedeutung von Akteuren ist, die nicht der Exekutive angehören. Wie wir in früheren Arbeiten gezeigt haben (Kriesi et al. 2012; Grande u. Kriesi 2014), sind öffentliche Debatten zu europäischen Themen durch spezifische Konfliktstrukturen und Akteurskonstellationen gekennzeichnet. Sie werden dominiert durch

\footnotetext{
${ }^{3}$ Wie die vergleichende Analyse von Hutter und Grande (2014) zeigt, hat die Bedeutung europäischer Themen in nationalen Wahlen zwar zugenommen, sie ist in den meisten Fällen aber noch immer unterdurchschnittlich.
} 
die Regierungen der EU-Mitgliedstaaten und supranationale Akteure, die den Kern einer proeuropäischen „Koalition“ bilden. In dieser Konstellation ist zu erwarten, dass die Polarisierung zwischen den Akteuren gering ist. Allerdings wurde die pro-europäische Koalition in der Eurokrise von verschiedenen Seiten herausgefordert. In den Schuldnerländern stießen die Austeritätspolitiken, die von der EU erzwungen wurden, auf Widerstand; in den Geberländern war es die Angst der Bürger um den dauerhaften Verlust von Hilfskrediten und Bürgschaften. In beiden Fällen eröffneten sich dadurch Mobilisierungschancen für opponierende politische Parteien und Bewegungen - und wo diese Chancen ergriffen wurden, führte dies zu einer stärkeren Polarisierung in der Debatte. Da wir in unserer Untersuchung aber nur Geberländer untersucht haben, erwarten wir insgesamt eine geringe Polarisierung (Polarisierungsthese).

Die Stärke der Polarisierung eines Konflikts hängt jedoch nicht nur von der Akteurskonstellation ab, sondern auch von der Art des Konflikts und dem Framing von Positionen. Aus der politikwissenschaftlichen Literatur ist bekannt, dass die Intensität politischer Konflikte unter zwei Bedingungen besonders stark ist: wenn es um redistributive Probleme geht (Lowi 1964) und wenn in Konflikten national-identitäre Begründungsmuster vorherrschen (Hooghe u. Marks 2009). Im Fall der Eurokrise scheinen beide Bedingungen zusammenzutreffen: „The Eurozone ,debt crisis` brings to the fore publicly across the region that ,what you get“ (redistribution) is strongly tied to ,who you are“ (identity)“ (Statham u. Trenz 2014: 14). Folglich wäre zu erwarten, dass in der Eurokrise nationalistische Frames an Bedeutung gewinnen und dies zu einer stärkeren Polarisierung führt (Framingthese).

Insgesamt sind unsere Erwartungen zu Ausmaß und Inhalt der Politisierung Europas in der Eurokrise zurückhaltender als die in der politikwissenschaftlichen Literatur dominierenden Thesen. Wir gehen zwar ebenfalls davon aus, dass die Salienz europäischer Themen in der Krise signifikant zugenommen hat, wir erwarten aber nicht, dass auch der Kreis der Akteure, die an diesen Debatten beteiligt waren, in gleichem Umfang ausgeweitet und die Beziehungen zwischen den Akteuren stärker polarisiert wurden. Wir vermuten im Gegenteil, dass die Eurokrise die Stellung der politischen Exekutiven, insbesondere der nationalen Regierungen und der supranationalen Institutionen, gestärkt hat. Schließlich erwarten wir, dass die Politisierung der Eurokrise zu einer Intensivierung jener nationalistischen und kulturell-identitärer motivierten Konflikte führte, die wir in unseren früheren Studien zum Wandel politischer Konfliktstrukturen in westeuropäischen Ländern identifiziert haben (Kriesi et al. 2008, 2012). 


\section{Forschungsdesign und Methoden}

Unsere Studie basiert auf Originaldaten, die wir von nationalen Printmedien erhoben haben. Die Berichterstattung in Massenmedien ist für die Analyse des politischen Konflikts eine unverzichtbare Quelle (Koopmans u. Statham 2010b; Statham u. Trenz 2013a). Die Medienberichterstattung ermöglicht nicht nur, die Sichtbarkeit der Akteure in einem Konflikt zu untersuchen, sondern auch die Themen, die sie ansprechen, die Positionen, die sie zu ihnen einnehmen, und die Begründungen, die sie zur Rechtfertigung ihrer Positionen vorbringen. Andere Quellen (z.B. Parteiprogramme, Parlamentsdebatten oder Expertenbefragungen) decken in der Regel nur einzelne Aspekte ab und können kein Gesamtbild eines politischen Konflikts liefern.

Wir analysieren die öffentlichen Debatten über alle wichtigen Integrationsschritte in sechs westeuropäischen Ländern (Deutschland, Frankreich Großbritannien, Österreich, Schweden und die Schweiz). Von unserer Untersuchung haben wir bewusst Länder ausgeschlossen, die erst in den 2000er Jahren Mitglied der EU wurden, weil in diesen nur eine kleine Zahl von Integrationsdebatten stattgefunden haben, und insbesondere die Debatten um den Maastrichter Vertrag und den Verfassungsvertrag, von denen behauptet wird, dass sie bislang am stärksten politisiert waren, dort nicht in gleicher Weise beobachtet werden können. Die von uns ausgewählten Länder unterscheiden sich im Hinblick auf wichtige Kontextfaktoren, die für die Stärke und den Zeitpunkt der Politisierung von Bedeutung sein können und es somit ermöglichen, die Gültigkeit der von uns formulierten Hypothesen zu überprüfen. Am wichtigsten ist, dass sich die sechs Länder in Bezug auf die Dauer und den Umfang ihrer Integration in der EU unterscheiden. Mit Frankreich und Deutschland enthält die Länderauswahl zwei Gründungsmitglieder der Europäischen Gemeinschaften; Großbritannien war in der ersten Gruppe der Beitrittsländer (Beitritt 1973); und Österreich und Schweden wurden in der dritten Erweiterungsrunde 1995 EU-Mitglieder. Drei der Länder (Deutschland, Frankreich und Österreich) sind Mitglieder der Eurozone, während sich Großbritannien und Schweden ausdrücklich gegen eine Mitgliedschaft entschieden haben. Um einen zusätzlichen Maßstab für die Intensität des politischen Konflikts in Integrationsfragen zu bekommen, haben wir die Schweiz in unsere Untersuchung aufgenommen. Die öffentlichen Debatten und nationalen Referenden über europäische Themen in der Schweiz liefern wichtige Erkenntnisse über die Politisierung von Mitgliedschaftskonflikten in einem Land, in dem der Beitritt in die EU gescheitert ist. Da 
unsere Länderauswahl jedoch keines der von der Eurokrise besonders stark betroffenen Schuldnerländer enthält, ist die Generalisierbarkeit unserer Untersuchungsergebnisse für die Gesamtheit der EU-Mitgliedstaaten begrenzt.

Unsere Daten umfassen die öffentlichen Debatten über alle formellen Integrationsschritte seit den frühen 1970er Jahren. Genauer gesagt konzentrieren wir uns auf die Debatten über erfolgreiche oder gescheiterte Vertragsreformen nach der Entscheidung über die Römischen Verträge im Jahr 1957 (das sind: die Einheitliche Europäische Akte, Maastricht, Amsterdam, Nizza, der Verfassungsvertrag und Lissabon) und auf alle Erweiterungsentscheidungen (die Norderweiterung mit dem Beitritt Großbritanniens, Irlands und Dänemarks; die beiden Süderweiterungen mit den Beitritten Portugals, Spaniens und Griechenlands, die „EFTA-Erweiterung“ 1995 und die beiden „Osterweiterungen“ 2004 und 2007). Wir haben außerdem die Debatte über den EU-Beitritt der Türkei in die Untersuchung einbezogen, da diese in einigen EU-Mitgliedstaaten stark politisiert war. Außerdem haben wir acht länderspezifische Debatten aus Österreich, Schweden und der Schweiz in unsere Untersuchung aufgenommen. Diese Debatten betrafen wichtige Entscheidungen des jeweiligen Landes in Bezug auf die EG/EU bzw. seine Integration in den europäischen Binnenmarkt oder die Wirtschafts- und Währungsunion. Zu diesen Debatten zählt das Freihandelsabkommen Österreichs und der Schweiz mit der EG in den frühen 1970er Jahren; die Mitgliedschaft Österreichs, Schwedens und der Schweiz im Europäischen Wirtschaftsraum (EWR) in den frühen 1990er Jahren; die beiden bilateralen Verträge zwischen der Schweiz und der EU und die schwedische Debatte über den Beitritt zur Eurozone zu Beginn der 2000er Jahre. Insgesamt basiert unsere Analyse auf 86 nationalen öffentlichen Debatten über 18 verschiedene Integrationsschritte.

Unsere Untersuchungseinheit, der Integrationsschritt, ist kein singuläres Ereignis, sondern erstreckt sich über einen längeren Zeitraum. Dies gilt insbesondere für die Erweiterungsentscheidungen, die von der ersten Entscheidung über die Aufnahme von Beitrittsverhandlungen bis zur formellen Ratifizierung der Mitgliedschaftsvereinbarung mehrere Jahre in Anspruch nehmen können. Deshalb wäre es irreführend, nur die letzte Phase der jeweiligen Integrationsdebatte, also die entscheidende intergouvernementale Entscheidung oder den Abschluss der nationalen Ratifizierungsverfahren, zu untersuchen. Aus diesem Grund unterteilten wir jeden Integrationsschritt in eine Reihe von wichtigen formellen TeilEntscheidungen und erhobenen Daten über die öffentliche Debatte für jede dieser Phasen. Diese „kritischen Phasen“ sind (a) die Einleitung eines Integrationsschritts (z.B. der formelle 
Antrag eines Landes auf Mitgliedschaft oder ein EU-Gipfel), (b) die Reaktion der Europäischen Kommission (im Falle von Erweiterungsschritten), (c) der Beginn der Verhandlungen, (d) die Unterzeichnung eines Vertrags, und (e) die endgültige nationale Entscheidung über Annahme oder Ablehnung (entweder durch das nationale Parlament oder durch ein Referendum). Für jede dieser Phasen haben wir zwei Wochen vor und eine Woche nach dem Datum der jeweiligen Entscheidung alle Artikel erfasst, die sich mit dem betreffenden Integrationsschritt befassten.

Wir stützen uns auf Artikel aus einer nationalen Qualitätstageszeitung pro Land: Die Presse (Österreich), The Times (Großbritannien), Le Monde (Frankreich), Süddeutsche Zeitung (Deutschland) und Neue Zürcher Zeitung (Schweiz). Da wir nicht nur am Umfang der Presseberichterstattung in einer Debatte interessiert sind, sondern auch an der Intensität des Konflikts zwischen politischen Akteuren, haben wir für die Kodierung der Artikel eine aufwendige Methode der quantitativen Inhaltsanalyse verwendet: die kernsatzbasierte Inhaltsanalyse. Diese Methode wurde ursprünglich von Kleinnijenhuis und seinen Kollegen entwickelt (u.a. Kleinnijenhuis u. Pennings 2001) und von uns in mehreren Projekten für die Analyse von politischen Konflikten und Konfliktstrukturen in westeuropäischen Ländern fruchtbar gemacht (Kriesi et. al 2008, 2012; Hutter et al. i.E.). Bei dieser Methode ist die Analyseeinheit weder ein einziges Schlagwort noch der ganze Artikel, sondern ein "Kernsatz", der aus einer logischen Beziehung zwischen einem „Subjekt“ und einem „Objekt“ besteht. Für die vorliegende Studie haben wir nur solche Kernsätze in einem Artikel berücksichtigt, die sich thematisch auf Europa beziehen. Um die Art der Beziehung zwischen Akteuren zu bestimmen, benutzen wir eine Skala von -1 bis +1 mit drei Zwischenpositionen, wobei -1 eine Kritik oder Ablehnung eines Akteurs angibt und +1 eine positive Aussage. Neben den in einem Artikel erwähnten Akteuren, Themen und Positionierungen haben wir auch die Frames kodiert, welche die Akteure zur Rechtfertigung ihrer Positionen verwenden. Wir kodierten diese Frames ebenfalls auf der Ebene der Kernsätze. Da Akteure mitunter mehrere Argumente zur Begründung ihrer Position anführen, haben wir bis zu drei solcher Frames für jeden Akteur-Themen-Satz erlaubt. ${ }^{4}$ Insgesamt basiert unsere Analyse auf ca. 7.200 Artikeln, ca. 60.000 Kernsätzen und ca. 24.000 Frames.

Zur Messung unseres Politisierungskonzepts verwenden wir für jede der drei Dimensionen einen gesonderten Indikator. Die Salienz einer Debatte messen wir mittels der durchschnittlichen Anzahl der an einem Tag kodierten Artikel. Je größer die Anzahl der

\footnotetext{
${ }^{4}$ Eine ausführliche Darstellung der von uns verwendeten Methode findet sich bei Dolezal et al. (2012).
} 
Artikel mit einem Europabezug ist, desto höher ist die Salienz. Die Reichweite des

Akteurskreises messen wir anhand des Anteils der Aussagen von Akteuren, die nicht Mitglied der jeweiligen nationalen Regierung sind (z.B. Parlamentarier, zivilgesellschaftliche Akteure, Experten). Je größer der Anteil dieser Aussagen in Relation zum Anteil von

Regierungsakteuren ist, desto größer ist die Akteursexpansion. Zur Messung der Polarisierung verwenden wir den von Taylor und Hermann (1971) entwickelten Indikator der ideologischen Polarisierung (zur genauen Berechnung Kriesi et al. 2008, S. 364). Dieser Indikator bewegt sich auf einer Skala von 0 bis 1, wobei er mit zunehmender Polarisierung ansteigt.

\section{Die Gesamtentwicklung der Politisierung in der Debatte um die Eurokrise}

Wie stark war die Politisierung in der öffentlichen Debatte um die Eurokrise? Abbildung 1 zeigt die Entwicklung der Politisierung im Zeitraum von Dezember 2009 bis März 2012 für alle sechs Länder zusammengenommen. Eine solche integrierte europäische Debatte ist am besten geeignet, Vergleiche zwischen einzelnen Integrationsschritten vorzunehmen. Um die Interpretation der Ergebnisse zu erleichtern, haben wir die Politisierung in einem Bereich von 0 bis 1 standardisiert, wobei 1 dem monatlichen Maximum entspricht und 0 dem monatlichen Minimum. Die Abfolge der monatlichen Salienzen wurde geglättet, indem wir gleitende Mittelwerte verwendeten (ungewichtete Mittelwerte - t, t-1, t + 1). Wie das Schaubild zeigt, stieg die Politisierung mit der Schuldenkrise Griechenlands im Frühjahr 2010 an. ${ }^{5}$ Im Dezember 2009 hatte die Besorgnis über die Verschuldung einiger EU-Mitgliedstaaten zugenommen und die im Herbst 2009 neu gewählte griechische Regierung hatte zugestanden, dass der öffentliche Schuldenstand des Landes manipuliert worden war und die tatsächliche Verschuldung mit 113 Prozent des Bruttoinlandsprodukts nahezu das Doppelte des in der Eurozone zulässigen Höchstwerts von 60 Prozent betrug. Als die griechische Regierung im Februar 2010 eine Reihe von Sparmaßnahmen zur Eindämmung des Defizits beschloss, verschärfte sich die Debatte über die Euro-Krise. Die Politisierung erreichte im Mai 2010 einen ersten Höhepunkt mit der Entscheidung des Europäischen Rats über das erste Hilfspaket für Griechenland und die Einrichtung eines temporären Rettungsfonds für Länder mit Zahlungsschwierigkeiten (EFSF). Im Sommer 2010 beruhigte sich die Debatte, sie erreichte aber mit dem Hilfspaket für Irland im November 2010 einen weiteren Höhepunkt.

\footnotetext{
${ }^{5} \mathrm{Zu}$ Ursachen und Verlauf der Eurokrise und den politischen Rettungs- und Reformentscheidungen in dieser Phase siehe Eichengreen (2012), Featherstone (2011), Hall (2012), Lane (2012), Scharpf (2011) und Sinn (2012).
} 


\section{[Abbildung 1]}

Zu Beginn des nächsten Jahres schwächte sich die Debatte erneut ab, ohne jedoch vollständig abzuklingen. Die Politisierung stieg mit dem Hilfspaket für Portugal im Mai 2011 wieder an und sie intensivierte sich mit dem zweiten Hilfspaket für Griechenland im Juli 2011 weiter. Ihren Gipfel erreichte die Politisierung im Herbst 2011, als die umfassende Strategie diskutiert wurde, die der Europäische Rat auf seinem Gipfeltreffen im Dezember 2011 beschloss. Dazu zählten die Reform des „Stabilitäts- und Wachstumspakts“ der EU (mit der Fiskalunion und dem „Sixpack“) und die Umwandlung des EFSF in den permanenten Europäischen Stabilitätsmechanismus (ESM). Im Anschluss an diese Entscheidungen ging die Politisierung wieder deutlich zurück, was nicht zuletzt auch daran lag, dass Schlüsselentscheidungen in der Euro-Rettungspolitik seit dem Sommer 2012 zunehmend von der EZB und nicht mehr von den Staats- und Regierungschefs der EU-Mitgliedstaaten getroffen wurden. Obwohl unsere Daten also nur die Entwicklung der Politisierung bis Ende März 2012 erfassen, decken sie die kritischsten Phasen in der Entwicklung und die wichtigsten Entscheidungen auf europäischer Ebene bis zum Ende des Jahres 2014 ab.

Ein kurzer Blick auf die einzelnen Dimensionen der Politisierung zeigt, dass die Gesamtentwicklung eng der Entwicklung der Salienz folgt (siehe Abbildung 2). ${ }^{6}$ Dies liegt auch daran, dass die anderen beiden Dimensionen weniger stark variieren. An der Polarisierung änderte sich lange Zeit (abgesehen von einem kurzen Rückgang im Sommer 2010) wenig, während die Akteursexpansion mit dem ersten Hilfspaket für Griechenland einen ersten Höhepunkt erreicht und dann mit dem zweiten Hilfspaket wieder ansteigt. Dennoch wäre es falsch, die Politisierung der Eurokrise einfach mit ihrer Salienz gleichzusetzen. Der Blick auf die letzten von uns erfassten Phasen der Krise zeigt interessante Abweichungen der beiden anderen Dimensionen von der Gesamtentwicklung. Während die öffentliche Sichtbarkeit des Konflikts nach den Entscheidungen des Europäischen Rats im Dezember 2011 zurückging, stiegen sowohl die Akteursexpansion als auch die Polarisierung an. Die Akteursexpansion war bereits im Mai 2011 größer geworden, im Herbst aber wieder zurückgegangen, während die Polarisierung erst nach dem Dezember 2011 stark zunahm. Die Reformentscheidungen des Gipfels vom Dezember 2011 hatten also mit Blick auf die Politisierung der Eurokrise ein ambivalentes Ergebnis: Mit den Entscheidungen der Staats-

\footnotetext{
${ }^{6}$ Auch bei diesen beiden Dimensionen verwenden wir gleitende Mittelwerte der standardisierten Werte für die einzelnen Monate.
} 
und Regierungschefs ging zwar die öffentliche Sichtbarkeit des Konflikts deutlich zurück, aber seine Reichweite und Intensität nahm aber signifikant zu.

[Abbildung 2]

\section{Die Politisierung der Eurokrisen-Debatte im Vergleich}

Die öffentliche Debatte zur Eurokrise war offensichtlich politisiert und es war insbesondere die große Sichtbarkeit des Konflikts, die die Stärke der Politisierung bestimmte. Aber wie stark war diese Politisierung im Vergleich zu früheren Integrationsschritten? Im Folgenden vergleichen wir die Debatte über die Eurokrise mit jenen drei Debatten über Vertragsreformen, die den höchsten Politisierungsgrad aufweisen, dem Maastrichter Vertrag, dem Verfassungsvertrag und dem Vertrag von Lissabon. Außerdem präsentieren wir den Mittelwert aller Integrationsschritte (mit Ausnahme der Eurokrise), um einen zusätzlichen Anhaltspunkt für die Stärke der Politisierung zu geben. Wir untersuchen sowohl das Gesamtniveau der Politisierung und als auch die Werte für die einzelnen Dimensionen Salienz, Akteursexpansion und Polarisierung.

Ein solcher Vergleich ist für die letzten beiden Dimensionen unkompliziert, in Bezug auf die Salienz sind allerdings einige Erläuterungen zur Operationalisierung erforderlich. Wie oben bereits erwähnt, haben wir bei der Erfassung der Artikel zu den einzelnen Integrationsschritten nicht den gesamten Zeitraum von der Einleitung einer Vertragsreform bis zur nationalen Ratifizierung abgedeckt, sondern die Auswahl der Artikel auf kritische Phasen des Entscheidungsprozesses konzentriert. Die Salienz der Debatte wurde dann mit der durchschnittlichen Anzahl der kodierten Artikel pro ausgewähltem Tag operationalisiert. Abhängig von der Dauer einer Integrationsdebatte und der Anzahl der kritischen Phasen variiert die Zahl der ausgewählten Tage zwischen 74 und 273. Da die Debatte über die Eurokrise nicht in der gleichen Weise strukturiert war, haben wir nicht kritische Ereignisse bzw. Phasen ausgewählt, sondern den gesamten Zeitraum von Dezember 2009 bis März 2012 erfasst. Dieser Zeitraum erstreckt sich über 851 Tage, überschreitet also das Maximum für alle anderen Debatten bei weitem. Allerdings gab es in diesem Zeitraum an vielen Tagen keine Artikel über die Eurokrise. Lediglich an 474 Tagen erschien mindestens ein Artikel zur Eurokrise. Aufgrund dieses Unterschieds im Selektionsverfahren von Artikeln ist es denkbar, dass unser Indikator die Salienz der Eurokrise und damit das Gesamtniveau der Politisierung unterschätzt. Aus diesem Grund berechneten wir zusätzlich den Politisierungsindex (und seine 
einzelnen Komponenten) für die beiden kritischsten Phase in der Debatte, die Entscheidung über das erste Hilfspaket für Griechenland Anfang Mai 2010 und die Einigung auf den Fiskalpakt im Dezember 2011. ${ }^{7}$

Tabelle 1 zeigt die entsprechenden Werte für die einzelnen Debatten. Die Ergebnisse widersprechen den in der politikwissenschaftlichen Literatur formulierten Erwartungen und Behauptungen zur Politisierung der Eurokrise in wesentlichen Punkten. Die Debatte zur Eurokrise war zweifellos politisiert, aber der Durchschnittswert für den Politisierungsindex liegt nicht über, sondern sogar unter dem Durchschnittswert für alle anderen Integrationsdebatten. Selbst wenn wir die am stärksten politisierte Phase der EurokrisenDebatte nehmen, die Entscheidung über den Fiskalpakt, ist der Politisierungsindex $(0,64)$ niedriger als in der Debatte um den Maastrichter Vertrag $(0,70)$, wenngleich er über den Werten für den Verfassungsvertrag $(0,55)$ und für den Vertrag von Lissabon $(0,47)$ liegt. In dieser besonders kritischen Phase der Eurokrise lag die Politisierung zwar in der Spitzengruppe aller Integrationsdebatten, aber selbst in dieser Phase kann von einem Quantensprung im Ausmaß der Politisierung nicht die Rede sein.

\section{[Tabelle 1]}

Ein Blick auf die einzelnen Indikatoren bestätigt, dass die Debatte zwar sehr salient war, aber auch in dieser Dimension war die Eurokrisen-Debatte nicht herausragend. Der Mittelwert der Salienz $(0,72)$ liegt nur knapp über der durchschnittlichen Salienz aller anderen Integrationsschritte $(0,69)$ und knapp unter den Werten für den Verfassungsvertrag und den Vertrag von Lissabon. Da unsere Operationalisierung der Eurokrisen-Debatte die Salienz vermutlich unterschätzt, kommt den Werten für die beiden kritischen Phasen der Eurokrise besondere Bedeutung zu. Der Wert für das erste Hilfspaket für Griechenland $(0,81)$ liegt etwas höher als für die anderen Debatten, während der Wert für den Fiskalpakt $(1,56)$ klar herausragt. Er überschreitet sogar den höchsten Wert für alle anderen Integrationsschritte, den Maastrichter Vertrag $(1,51)$, geringfügig. Die Besonderheit der Eurokrise besteht offensichtlich nicht darin, dass sie eine signifikant größere öffentliche Sichtbarkeit hatte, sondern dass sie die durchschnittliche Salienz der normalen Integrationsschritte für einen außergewöhnlich langen Zeitraum erreichte und dass die Salienz zu einem außerordentlich kritischen Zeitpunkt besonders hoch war.

\footnotetext{
${ }^{7}$ Genauer gesagt haben wir die Politisierung für die drei Monate berechnet, die für die Debatte zu diesen beiden Schlüsselereignissen maßgeblich waren: im ersten Fall von April bis Juni 2010, im zweiten Fall von Oktober bis Dezember 2011.
} 
Allerdings waren die beiden anderen Dimensionen des Politisierungsindexes, die Akteursexpansion und die Polarisierung, durchschnittlich oder sogar unterdurchschnittlich und dies auch in den beiden kritischsten Phasen der Eurokrise. Zunächst ist eindeutig zu erkennen, dass die Eurokrise zu keiner signifikanten Ausweitung des Kreises der beteiligten Akteure führte. Der Durchschnittswert für diesen Indikator $(0,25)$ ist niedriger als der Mittelwert für alle Integrationsdebatten $(0,28)$ und er ist deutlich niedriger als der höchste Wert bei der Debatte über den Verfassungsvertrag $(0,46)$. Die Werte für die beiden am stärksten politisierten Phasen unterscheiden sich hiervon nur wenig. Die Werte für die Polarisierung bleiben sogar noch weiter hinter den gängigen Erwartungen zurück. Der Durchschnittswert für die Eurokrise $(0,16)$ liegt nicht nur deutlich unter dem Wert für die drei anderen stark politisierten Debatten, er liegt auch deutlich unter dem Mittelwert für alle anderen Integrationsschritte $(0,21)$. Das gilt auch für die beiden am stärksten politisierten Phasen. Die Debatte um die Reform des Stabilitäts- und Wachstumspakts war die am schwächsten polarisierte Debatte in unserer Untersuchung. Kurz gesagt: Die Debatte über die Eurokrise war zwar in ihrer Hochphase die Integrationsdebatte mit der größten öffentlichen Sichtbarkeit, aber sie war sicherlich nicht die breiteste und intensivste Integrationsdebatte. In dieser Hinsicht waren die Debatten über den Maastrichter Vertrag, den Verfassungsvertrag und den Vertrag von Lissabon deutlich stärker politisiert.

\section{Die Politisierung der Eurokrisen-Debatte im internationalen Vergleich}

Da die sechs von uns untersuchten Länder in unterschiedlichem Ausmaß in die Eurozone bzw. die EU integriert und damit von der Eurokrise betroffen sind, erwarten wir auch signifikante Unterschiede zwischen diesen Ländern im Grad der Politisierung. Tabelle 2 und Abbildung 3 zeigen die Politisierung der Eurokrise für jedes der sechs Länder. Die Ergebnisse entsprechen weitgehend unseren Erwartungen. Der Politisierungsindex war in den drei Mitgliedstaaten der Eurozone (Deutschland, Frankreich und Österreich) deutlich höher als in den drei anderen Ländern. Bei weitem am stärksten politisiert war die Debatte in Deutschland $(0,61)$, gefolgt von Österreich $(0,34)$ und Frankreich $(0,24)$. Dies mag angesichts der Tatsache, dass Deutschland in der Eurokrise eine Schlüsselrolle spielte, nicht verwundern. Bedenkt man allerdings, (a) dass das Politisierungsniveau des Integrationsprozesses in Deutschland in der Vergangenheit sehr niedrig war (Hutter u. Grande 2014), (b) dass in dem von uns untersuchten Zeitraum keine nationale Wahl stattfand und es (c) (noch) keine 
relevante rechtspopulistische oder euroskeptische Partei auf nationaler Ebene gab, so ist das Ergebnis doch höchst bemerkenswert.

[Tabelle 2 und Abbildung 3]

Die außergewöhnlich starke Politisierung der Eurokrise in Deutschland zeigt sich auch, wenn man die beiden Höhepunkte der Krise gesondert betrachtet und vergleicht (Abbildung 3). Insbesondere die Debatte um den Fiskalpakt hat einen extrem hohen Wert $(1,61)$, der die Werte in den anderen Ländern weit überragt. Im Vergleich dazu liegt in Frankreich, dem zweiten Geberland, das im Zentrum der Rettungspolitik stand, nicht nur der Gesamtwert der Politisierung deutlich niedriger $(0,24)$, sondern auch der Wert für die kritischste Phase.

Damit ist jedoch nicht gesagt, dass die Debatte nur in den Mitgliedstaaten der Eurozone politisiert war. Unsere Ergebnisse zeigen, dass die Debatte über die Eurozone und sogar über die EU hinausreichte. Auch in Schweden und der Schweiz beobachten wir eine moderate Politisierung. In der Schweiz erreichte die Politisierung in der kritischsten Phase der Eurokrise sogar einen höheren Wert als in Frankreich und Österreich. Das mag darin begründet liegen, dass die Schweiz als wichtiges internationales Finanzzentrum und bedeutender Handelspartner der EU sehr besorgt über die Auswirkungen der Eurokrise auf die eigene Währung war.

Allerdings fällt auf, dass die Eurokrise in Großbritannien kaum politisiert war. Der Gesamtwert beträgt nur 0,07, was nicht zuletzt eine Folge der sehr niedrigen Sichtbarkeit der Debatte ist. Großbritannien ist zwar kein Mitglied der Eurozone, aufgrund der exponierten Stellung des Landes in der EU und als internationales Finanzzentrum einerseits, der Tatsache, dass die Europapolitik in der Vergangenheit immer wieder höchst kontrovers zwischen den britischen Parteien war und zu einer starken Politisierung von Wahlen führte (Hutter u. Grande 2014) anderseits, wäre eine stärkere Politisierung der Eurokrise zu erwarten gewesen. Die Sonderstellung Großbritanniens in der Eurokrisen-Debatte fällt insbesondere in den beiden kritischsten Phasen auf, die kaum politisiert waren. Das erste Hilfspaket für Griechenland und der drohende „Grexit“ waren in Großbritannien kein Thema und die Debatte um den Fiskalpakt wurde von der Exekutive dominiert. Folglich war die Polarisierung nur gering, was angesichts der Tatsache, dass die britische Regierung in dieser Phase der Eurokrise eine in den anderen Ländern höchst umstrittene Außenseiterposition einnahm, durchaus überrascht. Diese Ergebnisse legen nahe, dass der starke Anstieg der Politisierung Europas in Großbritannien in den vergangenen Jahren keine Folge der Eurokrise 
ist, sondern Ausdruck eines innenpolitischen Konflikts um die Mitgliedschaft in der EU, der weitgehend unabhängig von der Krise ist.

Eine detailliertere Analyse der drei Dimensionen der Politisierung in den sechs Ländern in Tabelle 2 bestätigt, dass das Gesamtniveau der Politisierung stark von der Salienz bestimmt wird. Die drei Mitgliedstaaten der Eurozone unterscheiden sich insbesondere hinsichtlich der Salienz der Debatte von den beiden anderen EU-Mitgliedstaaten. ${ }^{8}$ Vor allem Deutschland ragt mit einem Gesamtwert für die Salienz $(1,07)$ heraus. Im Hinblick auf die Akteursexpansion weisen zwei Länder überdurchschnittliche Werte auf: Deutschland $(0,36)$ und Österreich (0,31). In beiden Ländern beobachten wir eine breitere Beteiligung von nichtexekutiven Akteuren, von denen etwa ein Drittel aller Aussagen stammen. In allen anderen Ländern, auch in Frankreich, liegt ihre Beteiligung unter dem Durchschnitt der Integrationsdebatten. Im Vergleich dazu waren die Unterschiede zwischen den Ländern bei der Polarisierung gering und der höchste Wert (0,22 in Deutschland) lag nur geringfügig über dem Durchschnitt.

Aufgrund der sehr starken Politisierung der Eurokrise in Deutschland, dem wichtigsten Geberland, untersuchen wir diese Debatte detaillierter für die beiden kritischsten Phasen der Eurokrise. Die Ergebnisse in Tabelle 3 zeigen, dass wir in diesen beiden Phasen jene außergewöhnlich hohen Politisierungswerte finden, die in der Literatur für die Eurokrise insgesamt angenommen werden. Die Werte für die Politisierung des ersten griechischen Hilfspakets $(0,90)$ und für den Fiskalpakt $(1,61)$ liegen nicht nur weit über dem Durchschnitt der anderen Länder, sie übersteigen auch die Werte für die am stärksten politisierten Integrationsdebatten bei weitem. Die Debatte hatte in diesen beiden Phasen aber nicht nur eine extrem hohe öffentliche Sichtbarkeit (mit Werten von 1,25 für die erste und 2,40 für die zweite Phase), sie war auch außergewöhnlich breit. Mit 0,43 bzw. 0,45 erreichte die Akteursexpansion nahezu den Spitzenwert der Debatte um den Verfassungsvertrag. Die Polarisierung war in der Debatte um das erste Hilfspaket für Griechenland sehr hoch $(0,29)$, ging aber in der Debatte um den Fiskalpakt auf ein durchschnittliches Niveau zurück. Das hohe Politisierungsniveau in diesen beiden Phasen der Eurokrise weist auf ein außerordentlich hohes öffentliches Interesse und ein starkes Konfliktpotential in Deutschland hin, da die Politisierung weder durch ein nationales Referendum (wie im Fall des Maastrichter Vertrags

\footnotetext{
${ }^{8}$ Die Schweiz stellt angesichts des sehr hohen Anteils ausländischer Akteure an der Debatte einen Sonderfall dar und wird von uns hier nicht weiter betrachtet.
} 
in Frankreich) noch durch eine starke euroskeptische Partei im nationalen Parlament angetrieben wurde.

\section{[Tabelle 3]}

Insgesamt liefert die vergleichende Analyse der Eurokrisen-Debatte ein irritierendes Bild. Einerseits hatte die Debatte in den meisten Ländern eine große öffentliche Sichtbarkeit, andererseits wurde sie aber stark durch die Exekutiven dominiert und die Polarisierung zwischen den beteiligten Akteuren war dementsprechend relativ gering. Das Gesamtniveau der Politisierung war alles in allem nicht höher, sondern niedriger als in den wichtigsten Integrationsdebatten der 1990er und 2000er Jahre; und es war am höchsten in Deutschland, dem Land, in dem bei nationalen Wahlen und Integrationsdebatten zuvor das niedrigste Politisierungsniveau beobachtet wurde. Die Eurokrisen-Debatte ist ganz offensichtlich außergewöhnlich, aber dies auf eine andere Weise, als in einschlägigen Literatur behauptet. Wie kann man diese eigentümlichen Befunde erklären? Wir werden uns im Folgenden auf zwei Faktoren konzentrieren: die Akteurskonstellationen und die Bedeutung nationalistischer Frames. Von diesen beiden Faktoren wird vermutet, dass sie starken Einfluss auf jene beiden Politisierungsdimensionen haben, die in der Eurokrise nur schwach ausgeprägt waren, die Akteursexpansion und die Polarisierung.

\section{Akteure und Argumentationsstrategien in der Eurokrisen-Debatte}

In unserer Analyse der Akteursexpansion haben wir bereits auf den großen Anteil exekutiver Akteure in der Eurokrisen-Debatte verwiesen. Im Folgenden wollen wir diesen Aspekt vertiefen und die relative Bedeutung der einzelnen Akteurstypen herausarbeiten. Abbildung 4 zeigt die Verteilung der verschiedenen Akteurstypen im Vergleich mit dem Durchschnitt der anderen Integrationsdebatten. Es ist deutlich zu erkennen, dass die Eurokrisen-Debatte von zwei Akteursgruppen dominiert wurde: den europäischen Institutionen (Kommission, EuroGruppe, EZB und Rat) und den (ausländischen und nationalen) Regierungen. Auf die europäischen Institutionen entfällt mehr als ein Viertel aller Aussagen in der Debatte (26,6 Prozent), auf die Regierungen nahezu die Hälfte (49,4 Prozent). Unter den europäischen Institutionen dominieren die Exekutivorgane (Kommission, Euro-Gruppe, EZB, Rat), während das Europäische Parlament mit einem Anteil von 8,2 Prozent an den europäischen Beiträgen nur eine untergeordnete Rolle spielt. Auffällig ist auch, dass die Mehrzahl der Beiträge von nationalen Regierungen nicht von der Regierung des eigenen Landes kommt 
(10,9 Prozent der Gesamtaussagen), sondern von Regierungen anderer Länder (38,5 Prozent). Diese waren bereits in den anderen Integrationsdebatten stark vertreten, in der EurokrisenDebatte ragen sie jedoch klar heraus.

\section{[Abbildung 4]}

Alle anderen Akteure spielen in der Eurokrisen-Debatte nur eine untergeordnete Rolle. Das gilt insbesondere für die politischen Parteien, die an dieser Debatte weit weniger beteiligt waren als an anderen Integrationsdebatten. Auf Parteien entfielen insgesamt nur etwa ein Siebtel aller Beiträge, von denen die Mehrzahl nicht von den Parteien des eigenen Landes, sondern von ausländischen (7,8 Prozent) oder europäischen Parteien (1,0 Prozent) stammten. Besonders auffällig ist, dass sich nationale Parteien im Vergleich zu früheren Debatten signifikant weniger beteiligten.

Zusammengenommen ergeben sich aus dieser Analyse zwei wichtige Befunde. Zum einen zeigen sie, dass die Debatte um die Eurokrise außerordentlich stark europäisiert war und dies in beiden Dimensionen. Sie weist sowohl eine starke vertikale als auch eine ausgeprägte horizontale Europäisierung auf. Zum anderen bestätigt sie die ausgeprägte Exekutivdominanz der Eurokrisen-Debatte. Beides hatte Auswirkungen auf das spezifische Politisierungsprofil dieser Debatte. Die starke Europäisierung trug zweifellos zur hohen Salienz der Debatte bei, insbesondere in kleineren Ländern, die weniger stark von der Krise betroffen waren. Die Europäisierung der Debatte führte aber nicht zu einer stärkeren Politisierung, da dadurch die Exekutiven gestärkt wurden, und dies hatte zur Folge, dass die Akteursexpansion verringert und die Polarisierung reduziert wurden.

Welche Konflikte prägten die Politisierung der Eurokrise? Wir können diese Frage in diesem Beitrag nicht umfassend beantworten, wollen aber zur Abrundung unserer Ergebnisse einen Aspekt untersuchen, der im Zusammenhang mit der Politisierung des europäischen Integrationsprozesses in der Vergangenheit eine wichtige Rolle spielte: die Bedeutung von nationalistisch-identitären Argumentations- und Begründungsmustern (Frames). Die Politisierungsliteratur nimmt einen positiven Zusammenhang zwischen der Stärke solcher Frames und der Intensität der Politisierung an (Hooghe u. Marks 2009) und Hutter und Grande (2014) konnten diese Annahme für die Politisierung europäischer Themen in nationalen Wahlen auch bestätigen. Welche Bedeutung haben nationalistische Frames in der Eurokrisen-Debatte? 
In unserer Analyse der Positionierung der Akteure zu europäischen Themen haben wir drei Typen von Begründungsmustern unterschieden: kulturelle, ökonomische und utilitaristische Frames. Kulturelle Frames untergliederten wir in nationalistische und multikulturelle Frames, während wir bei utilitaristischen Frames zwischen politischen Effizienz-Argumenten und sonstigen utilitaristischen Frames unterschieden. ${ }^{9}$ Die Ergebnisse unserer Analyse finden sich in Tabelle 4. In dieser Tabelle vergleichen wir die Anteile der jeweiligen Frames in der Eurokrisen-Debatte mit dem Durchschnitt der anderen Integrationsschritte. Es zeigt sich, dass kulturelle Frames in der Eurokrisen-Debatte insgesamt eine weit geringere Rolle spielten als in den anderen Integrationsdebatten. Ihr Anteil betrug in der Eurokrise nur 15,6 Prozent, gegenüber 29,7 Prozent in den anderen Debatten. Es ist auch zu erkennen, dass der Anteil nationalistischer Frames in den Integrationsdebatten gering war (12,7 Prozent) und diese in der Eurokrisen-Debatte mit 7,0 Prozent von untergeordneter Bedeutung waren. In der Eurokrise dominierten eindeutig ökonomische Begründungsmuster (50,2 Prozent). Ihr Anteil lag in der Krise doppelt so hoch wie in den anderen Integrationsdebatten. Dies mag trivial erscheinen, war die Eurokrise doch in erster Linie eine ökonomische Krise, in der technische Fragen der Finanzierung und Re-Finanzierung von Staaten und Banken eine große Rolle spielten. Die Eurokrise löste jedoch nicht nur politische Konflikte innerhalb von Staaten aus, welche die vorhandene ökonomische Konfliktdimension verstärkten. Aufgrund ihrer länderübergreifenden Struktur erzeugte sie auch Interessenkonflikte zwischen Ländern, und die konfligierenden nationalen Interessen hätten durchaus mit nationalistisch-identitären Argumenten politisch gerechtfertigt werden können. Ein solches nationalistisches Framing war nicht nur in jenen Ländern denkbar, welche die unmittelbaren Leidtragenden eines „deutschen Europas“ (Beck 2012) waren, sondern auch in den Geberländern. Die Parole „Unser Geld für unsere Leute“, die die österreichische FPÖ auf dem Höhepunkt der Eurokrise plakatierte, war ein Beispiel für einen solchen Wohlfahrtschauvinismus.

Vor diesem Hintergrund ist die geringe Bedeutung nationalistischer Frames in der Eurokrisen-Debatte durchaus erklärungsbedürftig. Die Literatur zur Politisierung Europas und zur Transformation politischer Konfliktlinien in Westeuropa legt nahe, dass in diesem Zusammenhang populistische rechte Parteien eine zentrale Rolle spielen (Kriesi 2007; Hooghe u. Marks 2009; Kriesi et al. 2012). Wir haben aus diesem Grund den Anteil nationalistischer Frames an den Aussagen der verschiedenen Akteure, die an

\footnotetext{
${ }^{9}$ Eine ausführliche Erläuterung der Operationalisierung der einzelnen Frames findet sich in Dolezal et al. (2012).
} 
Integrationsdebatten beteiligt waren, ermittelt. Das Ergebnis ist in Tabelle 5 dargestellt. Darin zeigen wir den Anteil nationalistischer Frames differenziert nach Akteurstypen einerseits, nach Erweiterungsdebatten, Vertragsreformen und der Eurokrise andererseits. Die

Gesamtwerte lassen erkennen, dass nationalistische Frames vor allem in Erweiterungsdebatten (einschließlich des Beitritts des eigenen Landes) verwendet werden $(14,8)$, wenngleich ihr Anteil auch dort insgesamt gering ist. Die Werte bestätigen auch die Erwartung, dass solche Frames am häufigsten von rechten Parteien benutzt werden, allerdings liegt deren Wert für die Eurokrise (26,3) nur geringfügig über dem für Erweiterungsdebatten $(25,8)$ und dem Gesamtdurchschnitt $(23,5)$. Es ist auch zu erkennen, dass solche Frames in der Eurokrise darüber hinaus nur bei linken Parteien und im Europäischen Parlament eine nennenswerte Rolle spielten. Auffällig ist schließlich, dass nationalistische Frames von den Akteuren, die die Eurokrisen-Debatte dominierten, kaum verwendet wurden. Dies gilt nicht nur für die Akteure aus den anderen supranationalen Institutionen, sondern - mit Ausnahme der britischen Regierung - auch für die nationalen Regierungen. ${ }^{10}$

\section{[Tabelle 5]}

\section{Schlussfolgerungen}

Was folgt aus diesen Befunden für die politikwissenschaftliche Debatte um die politischen Folgen der Eurokrise? Offensichtlich konnten die Erwartungen, die zu Beginn dieses Beitrags formuliert wurden, nur zum Teil bestätigt werden. Dies gilt sowohl für die in der Literatur vorherrschenden Thesen, als auch für unsere eigenen theoretischen Erwartungen. Drei Ergebnisse verdienen abschließend besondere Erwähnung.

Erstens bestätigen unsere Ergebnisse die Annahme, dass die Eurokrise zu einer substantiellen Europäisierung der öffentlichen Debatte geführt und damit die Herausbildung einer europäischen Öffentlichkeit beschleunigt hat (Koopmans u. Statham 2010a; Risse 2010, 2014b). Die öffentliche Debatte in der Euro-Krise war tatsächlich eine europäische Debatte mit einer großen Sichtbarkeit, auch wenn die Salienz insgesamt nicht höher war als in den anderen stark politisierten Integrationsdebatten. Im Fall der Eurokrise hatte diese

\footnotetext{
${ }^{10}$ Eine differenziertere Analyse der Argumentations- und Begründungsstrategien nationaler Regierungen, deren Ergebnisse wir hier aus Platzgründen nicht präsentieren können, zeigt, dass die britische Regierung in allen Integrationsdebatten überdurchschnittlich häufig von nationalistischen Frames Gebrauch machte. In der Eurokrise bestanden nicht weniger als 36 Prozent ihrer Begründungsmuster aus nationalistischen Frames (bei einem Durchschnittswert für nationale Regierungen von 8,1 Prozent).
} 
Europäisierung sowohl eine ausgeprägte vertikale als auch eine außergewöhnlich starke horizontale Dimension. Sie war, wie von uns erwartet, in den drei Ländern der Eurozone besonders intensiv, aber sie erstreckte sich auch auf jene Länder, die nicht Mitglieder der Eurozone waren. Unsere beiden Hypothesen zur Salienz und zur Länderdifferenz konnten folglich bestätigt werden.

Zweitens konnten wir zeigen, dass die starke Europäisierung nicht - wie in der Literatur behauptet - zu einer Zunahme der Politisierung führte. Der politische Konflikt um die verschiedenen Hilfsmaßnahmen und institutionellen Reformen in der Eurokrise war zwar sehr salient, er blieb in den von uns untersuchten Ländern jedoch weitgehend auf die exekutiven Eliten begrenzt und dementsprechend war die Polarisierung gering. Eine substantielle Ausweitung des Konflikts auf die politischen Parteien und auf zivilgesellschaftliche Akteure fand nicht statt. Unsere Elitendominanzhypothese und unsere Polarisierungshypothese wurden also bestätigt. Eine Ausnahme bildete die Kontroverse um den Fiskalpakt in den Mitgliedstaaten der Eurozone, insbesondere in Deutschland. In dieser Phase war die Debatte tatsächlich außergewöhnlich stark politisiert. Es wäre aber irreführend, aus dieser singulären Phase der Krise allgemeine Schlussfolgerungen für die Eurokrise insgesamt zu ziehen. Dies ist selbst für Deutschland, wo die Politisierung am stärksten und die Debatte am breitesten war, nicht möglich, von den anderen untersuchten Ländern gar nicht zu reden.

Drittens, schließlich relativieren unsere Ergebnisse die Bedeutung redistributiver Konflikte für die Politisierung der Eurokrise. Auch wenn wir diese Konflikte selbst nicht untersucht haben, so sind unsere Ergebnisse in dieser Hinsicht doch aufschlussreich. Wie Gerhards und Lengfeld (2013) gezeigt haben, sind es „in erster Linie die kulturellen und nicht die sozioökonomischen Faktoren, die die Einstellung zur sozialen Gleichheit für Ausländer beeinflussen“ (Gerhards u. Lengfeld 2013: 199). Folglich würden wir erwarten, dass europäische redistributive Konflikte vor allem dann stark politisieren, wenn diese mit nationalistischen Begründungen verbunden werden. Gerade dies war in der Eurokrise jedoch nicht der Fall. Nationalistische Frames waren in der Eurokrise nur von sehr geringer Bedeutung, so dass unsere Framingthese nicht bestätigt werden konnte. Die Kritik an den Hilfsmaßnahmen und institutionellen Reformen wurde ganz überwiegend nicht mit nationalistisch-identitären Frames begründet. Dies lag nicht zuletzt an der insgesamt geringen Bedeutung der politischen Parteien in der Eurokrisen-Debatte. Nationalistische Argumentations- und Begründungsmuster wurden in Integrationsdebatten vor allem von 
politischen Parteien verwendet - und deren öffentliche Sichtbarkeit war in der EurokrisenDebatte nur äußerst gering. Dies dürfte erheblich dazu beigetragen haben, dass das Politisierungsniveau in den von uns untersuchten Geberländern trotz der ausgeprägten redistributiven Dimension der Eurokrise insgesamt niedriger war als in der Literatur erwartet.

Alles in allem hat die Eurokrise in den von uns untersuchten Ländern zwar politisiert, aber sie hat nicht zu einem beispiellosen neuen politischen Konfliktniveau geführt. Wenn es in der Debatte um die Eurokrise einen Quantensprung gegeben hat, dann in der Dominanz exekutiver Eliten. Bislang zumindest eröffnete die Eurokrise nicht den Weg zu einem „kosmopolitischen Europa“ (Beck u. Grande 2004) der Bürger und der zivilgesellschaftlichen Akteure. Stattdessen verstärkte sie die bürokratischen und technokratischen Deformationen des europäischen Projekts. Allerdings ist dieser Befund mit zwei Einschränkungen zu versehen. Zum einen können unsere Ergebnisse keine Gültigkeit für die Gruppe der Schuldnerländer beanspruchen. In diesen Ländern waren die Bürger durch die Austeritätspolitiken von Beginn an unmittelbar von den negativen Folgen der Krise betroffen - und entsprechend waren auch die politischen Konsequenzen der Krise sehr viel tiefgreifender als in den anderen Mitgliedstaaten der Eurozone. Zum anderen können wir nicht davon ausgehen, dass die Eurokrise bereits vorbei ist - und damit auch die Geschichte ihrer Politisierung. Es ist nicht ausgeschlossen, dass durch die von den politischen Eliten gewählte Krisenbearbeitungsstrategie zwar kurzfristig das Politisierungsniveau zumindest in den Geberländern niedrig gehalten werden konnte (Schimmelfennig 2013), dass aber gerade der dadurch ausgelöste „Sog der Technokratie“ (Habermas 2013) längerfristig einen neuen Politisierungsschub auch in den Geberländern auslöst. Die „gekaufte Zeit“ (Streeck 2013) hätte dann einen hohen politischen Preis. 


\section{Literatur}

Beck, Ulrich. 2000. The Cosmopolitan Perspective. Sociology of the Second Age of Modernity. British Journal of Political Science 51:79-105.

Beck, Ulrich. 2012. Das deutsche Europa: Neue Machtlandschaften im Zeichen der Krise. Berlin: Suhrkamp.

Beck, Ulrich, und Edgar Grande. 2004. Das kosmopolitische Europa. Frankfurt a.M.: Suhrkamp.

De Wilde, Pieter. 2011. No Polity for Old Politics? A Framework for Analyzing the Politicization of European Integration. Journal of European Integration 33:559-575.

De Wilde, Pieter, und Michael Zürn. 2012. Can the Politicization of European Integration be Reversed? Journal of Common Market Studies 50:137-53.

Dolezal, Martin, Swen Hutter, und Bruno Wüest. 2012. Exploring the new cleavage across arenas and public debates: design and method. In Political Conflict in Western Europe, Hrsg. Hanspeter Kriesi, Edgar Grande, Martin Dolezal, Marc Helbling, Dominic Höglinger, Swen Hutter und Bruno Wüest, 36-63. Cambridge: Cambridge University Press.

Drewski, Daniel. 2015. Has there been a European Public Discourse on the Euro Crisis? A Systematic Content Analysis of Newspaper Editorials on the Euro Crisis in Germany and Spain. Working Paper No. 33. Berlin: FU Berlin, Institut für Soziologie.

Eichengreen, Barry. 2012. European Monetary Integration with Benefit of Hindsight. Journal of Common Market Studies 50:123-136.

Featherstone, Kevin. 2011. The Greek Sovereign Debt Crisis and EMU. A Failing State in a Skewed Regime. Journal of Common Market Studies 49:193-217.

Gerhards, Jürgen, und Holger Lengsfeld. 2013. Wir, ein europäisches Volk? Sozialintegration Europas und die Idee der Gleichheit aller europäischen Bürger. Wiesbaden: Springer VS.

Grande, Edgar, und Hanspeter Kriesi. 2014. The Re-Structuring of Political Conflict in Europe and the Politicization of European Integration. In European Public Spheres. Politics is Back, Hrsg. Thomas Risse, 190-223. Cambridge: Cambridge University Press.

Green-Pedersen, Christoffer. 2012. A Giant Fast Asleep? Party Incentives and the Politicisation of European Integration. Political Studies 60: 115-30. 
Guinaudeau, Isabelle, und Simon Persico. 2013. EU politicization through the lens of salience. How the EU enters the French, British and German electoral agenda (1986-2009). French Politics 11:143-68.

Habermas, Jürgen. 2013. Im Sog der Technokratie. Berlin: Suhrkamp.

Hall, Peter A. 2012. The Economics and Politics of the Euro Crisis. German Politics 21:355371.

Hix, Simon, und Christopher Lord. 1997. Political Parties in the European Union. Basingstoke: Palgrave.

Höglinger, Dominic. 2012. Struggling with the Intricate Giant - The Politicization of European Integration in Western Europe. Unveröffentlichte Dissertation: Universität Zürich.

Hooghe, Liesbet, und Gary Marks. 2009. A Postfunctional Theory of European Integration. From Permissive Consensus to Constraining Dissensus. British Journal of Political Science 39:1-23.

Hooghe, Liesbet, und Gary Marks. 2012. Politicization. In The Oxford Handbook of the European Union, Hrsg. Erik Jones, Anand Menon und Stephen Weatherill, 840-854. Oxford: Oxford University Press.

Hutter, Swen, und Edgar Grande. 2014. Politicizing Europe in the National Electoral Arena. A Comparative Analysis of Five West European Countries, 1970-2010. Journal of Common Market Studies 52:1002-18.

Hutter, Swen, Edgar Grande, und Hanspeter Kriesi (Hrsg.). Politicizing Europe. Towards Constraining Dissensus? Cambridge: Cambridge University Press. (im Erscheinen).

Hutter, Swen, und Alena Kerscher. 2014. Politicizing Europe in Hard Times. Conflicts over Europe in France in a Long-term Perspective, 1974-2012. Journal of European Integration 36:267-282.

Kleinnijenhuis, Jan, und Paul Pennings. 2001. Measurement of party positions on the basis of party programmes, media coverage and voter perceptions. In Estimating the Policy Positions of Political Actors, Hrsg. Michael Laver, 162-182. London: Routledge.

Koopmans, Ruud. 2007. Who inhabits the European public sphere? Winners and losers, supporters and opponents in Europeanised political debates. European Journal of Political Research 46:183-210. 
Koopmans, Ruud. 2010. Winners and Losers, Supporters and Opponents in Europeanized Public Debates. In The Making of a European Public Sphere, Hrsg. Ruud Koopmans und Paul Statham, 70-97. Cambridge: Cambridge University Press.

Koopmans, Ruud, und Paul Statham (Hrsg.). 2010a. The Making of a European Public Sphere. Media Discourse and Political Contention. Cambridge: Cambridge University Press.

Koopmans, Ruud, und Paul Statham. 2010b. Theoretical Framework, Research Design, and Methods. In The Making of a European Public Sphere. Media Discourse and Political Contention, Hrsg. Ruud Koopmans und Paul Statham, 34-62. Cambridge: Cambridge University Press.

Kriesi, Hanspeter. 2007. The Role of European Integration in National Election Campaigns. European Union Politics 8: 83-108.

Kriesi, Hanspeter. 2014. The political consequences of the economic crisis in Europe: electoral punishment and popular protest. In Mass politics in tough times. Opinions, votes, and protest in the Great Recession, Hrsg. Nancy Bermeo und Larry M. Bartels, 297-333. Oxford: Oxford University Press.

Kriesi, Hanspeter, und Edgar Grande. 2014. The Europeanization of the National Political Debate. In Democratic Politics in a European Union under Stress, Hrsg. Olaf Cramme und Sara B. Hobolt, 67-86. Oxford: Oxford University Press.

Kriesi, Hanspeter, Edgar Grande, Martin Dolezal, Marc Helbling, Dominic Höglinger, Swen Hutter, und Bruno Wüest (Hrsg.). 2012. Political Conflict in Western Europe. Cambridge: Cambridge University Press.

Kriesi, Hanspeter, Edgar Grande, Romain Lachat, Martin Dolezal, Simon Bornschier, und Timotheos Frey (Hrsg.). 2008. West European Politics in the Age of Globalization. Cambridge University Press.

Lane, Philip R. 2012. The European Sovereign Debt Crisis. Journal of Economic Perspectives 26:49-68.

Leuffen, Dirk, Berthold Rittberger, und Frank Schimmelfennig. 2013. Differentiated Integration. Explaining Variation in the European Union. Basingstoke: Palgrave.

Lowi, Theodore. 1964. American Business, Public Policy, Case Studies and Political Theory. World Politics 16:677-715. 
Rauh, Christian, und Michael Zürn. 2014. Zur Politisierung der EU in der Krise. In Krise der europäischen Vergesellschaftung, Hrsg. Martin Heidenreich, 121-145. Wiesbaden: Springer.

Risse, Thomas. 2010. A Community of Europeans? Transnational Identities and Public Spheres. Ithaca: Cornell University Press.

Risse, Thomas (Hrsg.). 2014a. European Public Spheres. Politics is Back - Introduction. In European Public Spheres: Politics is Back, Hrsg. Thomas Risse, 1-25. Cambridge: Cambridge University Press.

Risse, Thomas (Hrsg.). 2014b. European Public Spheres. Politics is Back. Cambridge: Cambridge University Press.

Scharpf, Fritz W. 2011. Monetary Union, Fiscal Crisis and the Preemption of Democracy. MPIfG Discussion Paper 11/11. Köln: Max-Planck-Institut für Gesellschaftsforschung.

Schattschneider, Elmer E. 1957. Intensity, Visibility, Direction and Scope. American Political Science Review 51:933-942.

Schattschneider, Elmer E. [1960] 1975. The Semisovereign People. New York: Wadsworth Thomson Learning.

Schimmelfennig, Frank. 2010. The normative origins of democracy in the European Union: toward a transformationalist theory of democratization. European Political Science Review 2:211-33.

Schimmelfennig, Frank. 2013. European Integration in the Euro Crisis. The Limits of Postfunctionalism. Journal of European Integration 36:321-337.

Serricchio, Fabio, Myrto Tsakatika, und Lucia Quaglia. 2013. Euroscepticism and the Global Financial Crisis. Journal of Common Market Studies 51(1): 51-64.

Sinn, Hans-Werner. 2012. Die Target-Falle. München: Hanser.

Statham, Paul, und Hans-Jörg Trenz. 2013a. The Politicization of Europe: Contesting the Constitution in the mass media. London: Routledge.

Statham, Paul, und Hans-Jörg Trenz. 2013b. How European politicization can emerge through contestation. The Constitution case. Journal of Common Market Studies 51:965-980. 
Statham, Paul, und Hans-Jörg Trenz. 2014. Understanding the mechanisms of EU politicization. Lessons from the Eurozone crisis. Comparative European Politics. doi:10.1057//cep.2013.30.

Streeck, Wolfgang. 2013. Gekaufte Zeit: die vertagte Krise des demokratischen Kapitalismus. Berlin: Suhrkamp.

Zürn, Michael. 2012. Politisierung als Konzept der Internationalen Beziehungen. In Die Politisierung der Weltpolitik, Hrsg. Michael Zürn und Matthias Ecker-Ehrhardt, 7-35. Berlin: Suhrkamp.

Zürn, Michael, und Christian Rauh. 2014. Legitimationsprobleme im Früheuropäismus, Frankfurter Allgemeine Zeitung. 19.5.2014:6. 
Abbildung 1: Die Politisierung der Eurokrisen-Debatte im Zeitverlauf (Dezember 2009 März 2012) ${ }^{1}$

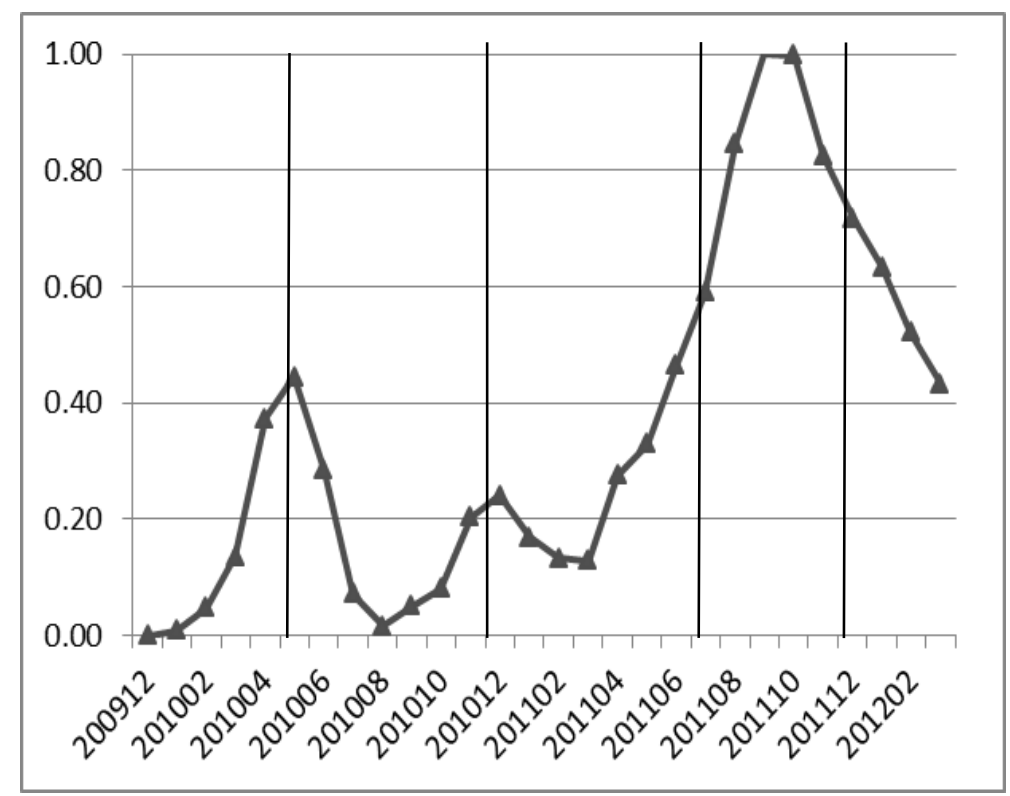

${ }^{1}$ Gleitender Durchschnitt der standardisierten Anzahl von Ereignissen (max.=1/min.=0).

${ }^{2}$ Drei Ereignisse markieren die drei „Spitzen“ der Politisierung: Das erste Hilfspaket für Griechenland im Mai 2010; das Hilfspaket für Irland im November 2010; und die Entscheidung über den Europäischen Fiskalpakt im Dezember 2011. 
Abbildung 2: Die Entwicklung der einzelnen Dimensionen des Politisierungsindex (gleitender Durchschnitt)
a) Salienz
b) Akteursexpansion und Polarisierung
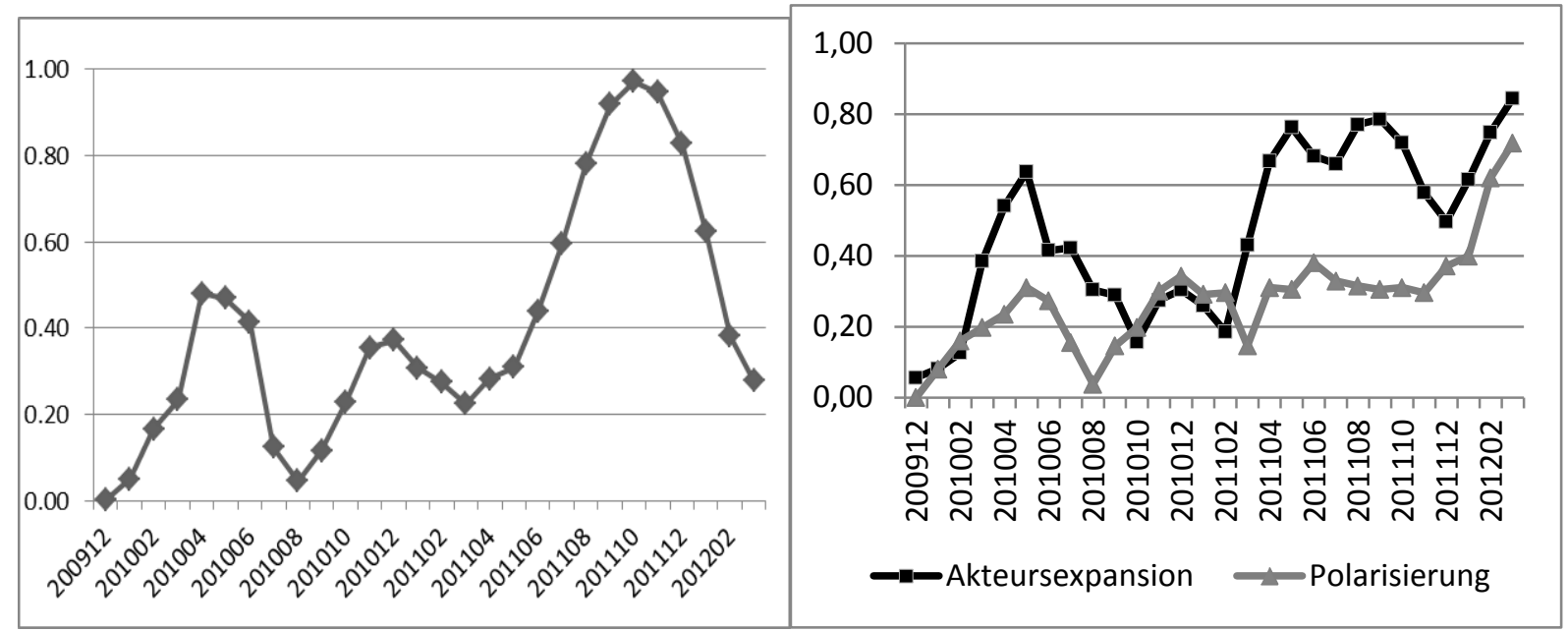
Tabelle 1: Die Politisierung der Eurokrise im Vergleich mit anderen Integrationsdebatten

\begin{tabular}{|c|c|c|c|c|}
\hline & Politisierungsindex & Salienz & Akteursexpansion & Polarisierung \\
\hline \multirow{2}{*}{\multicolumn{5}{|c|}{$\begin{array}{ll}\text { Alle } & \text { anderen } \\
\text { Integrationsschritte } & \end{array}$}} \\
\hline & & & & \\
\hline - Mittelwert & 0,35 & 0,69 & 0,28 & 0,21 \\
\hline - Minimum & 0,12 & 0,21 & 0,19 & 0,14 \\
\hline - Maximum & 0,70 & 1,51 & 0,46 & 0,36 \\
\hline \multirow{2}{*}{\multicolumn{5}{|c|}{$\begin{array}{l}\text { Ausgewählte } \\
\text { Integrationsschritte }\end{array}$}} \\
\hline & & & & \\
\hline - Maastrichter Vertrag & 0,70 & 1,51 & 0,24 & 0,22 \\
\hline - Verfassungsvertrag & 0,55 & 0,75 & 0,46 & 0,27 \\
\hline - Vertrag von Lissabon & 0,47 & 0,74 & 0,28 & 0,28 \\
\hline \multicolumn{5}{|l|}{ Eurokrise } \\
\hline - Mittelwert & 0,30 & 0,72 & 0,25 & 0,16 \\
\hline - Erstes Hilfspaket für & & & & \\
\hline Griechenland & 0,34 & 0,81 & 0,26 & 0,16 \\
\hline - Fiskalpakt & 0,64 & 1,56 & 0,27 & 0,14 \\
\hline
\end{tabular}


Tabelle 2: Die Politisierung der Eurokrisen-Debatte im internationalen Vergleich

\begin{tabular}{|c|c|c|c|c|}
\hline Land & $\begin{array}{l}\text { Politisierung } \\
\text { (Gesamtindex } \\
\text { ) }\end{array}$ & $\begin{array}{l}\text { Salienz } \\
\text { (Prozent) }\end{array}$ & $\begin{array}{l}\text { Nicht- } \\
\text { Regierungs- } \\
\text { akteure } \\
\text { (Prozent) }\end{array}$ & $\begin{array}{l}\text { Polarisierung } \\
\text { (Mittelwerte) }\end{array}$ \\
\hline Mitglieder der Eurozone: & & & & \\
\hline - Deutschland & 0,61 & 1,07 & 0,36 & 0,22 \\
\hline - Frankreich & 0,24 & 0,64 & 0,22 & 0,16 \\
\hline • Österreich & 0,34 & 0,73 & 0,31 & 0,15 \\
\hline EU-Mitgliedsstaaten außerhalb der & & & & \\
\hline Eurozone: & 0,07 & 0,19 & 0,17 & 0,20 \\
\hline $\begin{array}{l}\text { - Großbritannien } \\
\text { - Schweden }\end{array}$ & 0,11 & 0,29 & 0,20 & 0,17 \\
\hline $\begin{array}{l}\text { Andere Länder: } \\
\text { - Schweiz }\end{array}$ & 0,17 & 0,49 & 0,19 & 0,15 \\
\hline
\end{tabular}


Abbildung 3: Die Politisierung der Eurokrisen-Debatte in den kritischen Phasen im Vergleich

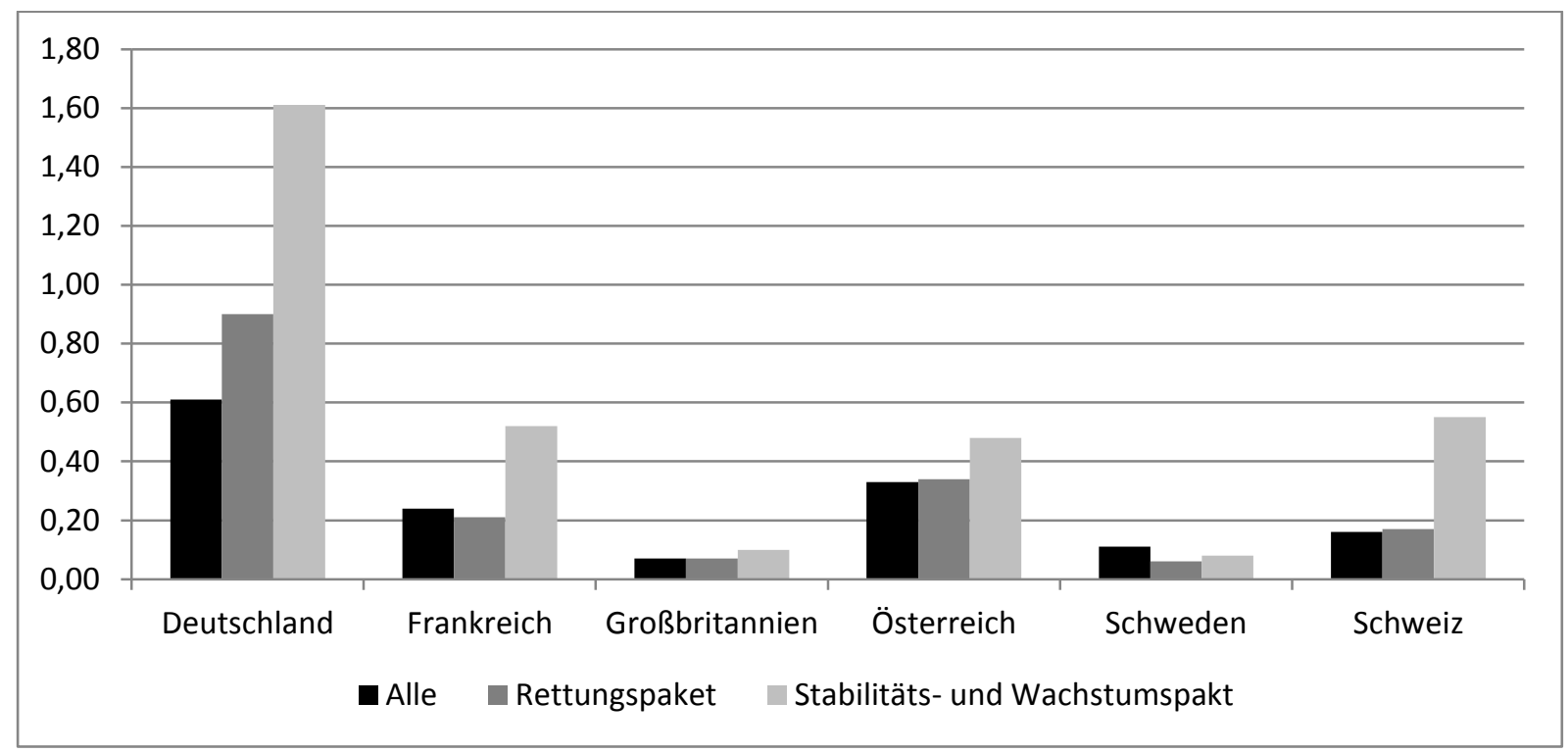


Tabelle 3: Die Politisierung der Eurokrisen-Debatte in Deutschland

\begin{tabular}{|l|c|c|l|c|}
\hline Zeitpunkt & $\begin{array}{l}\text { Politisierung } \\
\text { (gesamt) }\end{array}$ & $\begin{array}{l}\text { Salienz } \\
\text { (Prozent) }\end{array}$ & $\begin{array}{l}\text { Nicht- } \\
\text { Regierungs- } \\
\text { akteure } \\
\text { (Prozent) }\end{array}$ & $\begin{array}{l}\text { Polarisierung } \\
\text { (Mittelwerte) }\end{array}$ \\
\hline $\begin{array}{l}\text { Durchschnitt Eurokrise, alle } \\
\text { Länder }\end{array}$ & 0,30 & 0,72 & 0,25 & 0,16 \\
$\begin{array}{l}\text { Eurokrise gesamt } \\
\text { Erstes Rettungspaket } \\
\text { Griechenland }\end{array}$ & 0,61 & 1,07 & 0,36 & 0,22 \\
Stabilitäts- und Wachstumspakt & 1,61 & 1,25 & 0,43 & 0,29 \\
\hline
\end{tabular}


Abbildung 4: Akteure in der Eurokrisen-Debatte (in Prozent)

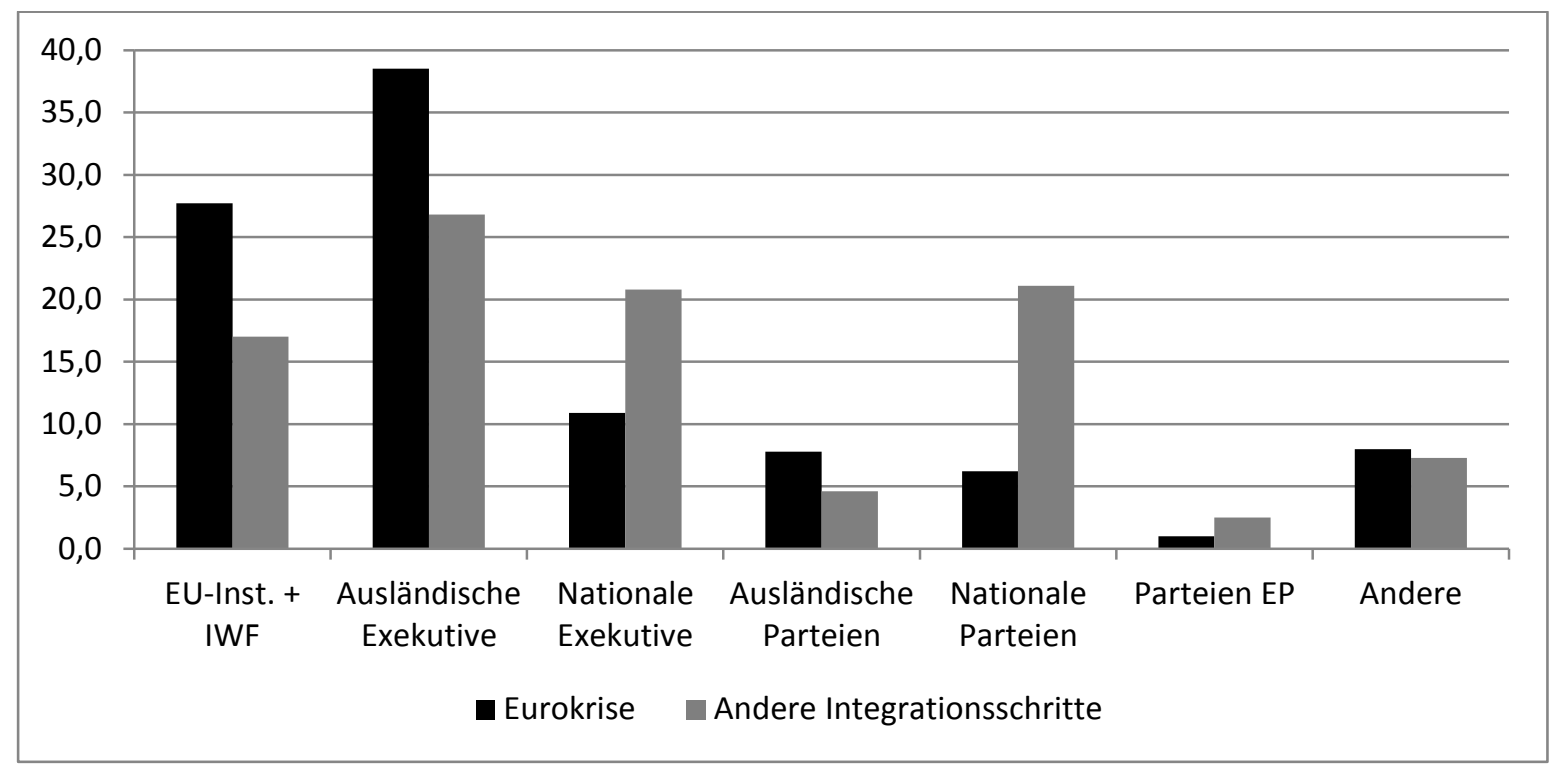


Tabelle 4: Das Framing der Eurokrise im Vergleich zu anderen Integrationsschritten (in Prozent)

\begin{tabular}{|l|cc|c|}
\hline & $\begin{array}{c}\text { Andere } \\
\text { Integrations- } \\
\text { schritte }\end{array}$ & Eurokrise & \\
\hline - Kulturell & 29,7 & 15,6 & 28,5 \\
- nationalistisch & 12,7 & 7,0 & 12,2 \\
- multikulturell & 17,0 & 8,6 & 16,3 \\
- Ökonomisch & 25,2 & 50,7 & 27,5 \\
- Utilitaristisch & 45,1 & 33,7 & 44,1 \\
- politische Effizienz & 18,7 & 21,2 & 18,9 \\
- andere utilitaristische & 26,4 & 12,5 & 25,2 \\
\hline Gesamt & 100,0 & 100,0 & 100,0 \\
n & 21.988 & 2.151 & 24.139 \\
\hline
\end{tabular}


Tabelle 5: Der Anteil nationalistischer Frames nach Akteurstyp

\begin{tabular}{|l|c|ccc|}
\hline & Gesamt & $\begin{array}{l}\text { Erweiter } \\
\text { ung }\end{array}$ & $\begin{array}{l}\text { Andere } \\
\text { Verträge }\end{array}$ & Eurokrise \\
\hline Nationale Parteien (rechte) & 23,5 & 25,8 & 20,2 & 26,3 \\
Nationale Parteien (linke) & 16,0 & 15,0 & 17,6 & 13,3 \\
Nationale Regierung & 15,0 & 17,6 & 11,0 & 8,1 \\
Europäische Parteien & 12,4 & 17,2 & 5,9 & 0,0 \\
Euro-Gruppe/EZB & 11,5 & 11,5 &. & 2,5 \\
Andere nationale Akteure & 10,5 & 8,8 & 12,8 & 11,0 \\
Andere & & & & \\
Akteure & & 4,0 & 4,2 & 0,0 \\
EU-Ministerrat & 4,0 & 5,1 & 0,7 & 1,0 \\
Nationale & 3,4 & & & \\
Interessengruppen & & 2,8 & 2,7 & 0,0 \\
Europäische Kommission & 1,4 & 1,4 & 1,5 & 0,0 \\
Europäisches Parlament & 1,0 & 1,7 & 0,0 & 13,3 \\
Andere & 14,7 & 13,5 & 16,2 & 6,6 \\
\hline Gesamt & 13,2 & 14,8 & 10,8 & 7,0 \\
\hline
\end{tabular}

